\title{
Rigid HYM Connections on Tautological Bundles over ALE Crepant Resolutions in Dimension Three
}

\author{
Anda DEGERATU ${ }^{\dagger}$ and Thomas WALPUSKI ${ }^{\ddagger}$ \\ $\dagger$ University of Freiburg, Mathematics Institute, Germany \\ E-mail: anda.degeratu@math.uni-freiburg.de \\ URL: https://home.mathematik.uni-freiburg.de/degeratu/ \\ $\ddagger$ Massachusetts Institute of Technology, Department of Mathematics, USA \\ E-mail: walpuski@mit.edu \\ URL: https://math.mit.edu/ walpuski/
}

Received June 02, 2015, in final form February 06, 2016; Published online February 15, 2016

http://dx.doi.org/10.3842/SIGMA.2016.017

\begin{abstract}
For $G$ a finite subgroup of $\operatorname{SL}(3, \mathbb{C})$ acting freely on $\mathbb{C}^{3} \backslash\{0\}$ a crepant resolution of the Calabi-Yau orbifold $\mathbb{C}^{3} / G$ always exists and has the geometry of an ALE non-compact manifold. We show that the tautological bundles on these crepant resolutions admit rigid Hermitian-Yang-Mills connections. For this we use analytical information extracted from the derived category McKay correspondence of Bridgeland, King, and Reid [J. Amer. Math. Soc. 14 (2001), 535-554]. As a consequence we rederive multiplicative cohomological identities on the crepant resolution using the Atiyah-Patodi-Singer index theorem. These results are dimension three analogues of Kronheimer and Nakajima's results [Math. Ann. 288 (1990), 263-307] in dimension two.
\end{abstract}

Key words: crepant resolutions; HYM connections

2010 Mathematics Subject Classification: 53C07; 14F05; 58J20

\section{Introduction}

Let $G$ be a finite subgroup of $\operatorname{SL}(n, \mathbb{C})$ and let $\pi: X \rightarrow \mathbb{C}^{n} / G$ be a crepant resolution. Often $X$ comes equipped with a collection of so-called tautological vector bundles $\mathscr{R}_{\rho}$ indexed by the set $\operatorname{Irr}(G)$ of irreducible representations of $G$. When $n=2$, Gonzalez-Sprinberg and Verdier [14] discovered that these vector bundles lie at the heart of the McKay correspondence. A geometrical interpretation of this result was given by Kronheimer and Nakajima [22] using asymptotically locally Euclidean (ALE) hyperkähler metrics on $X$ and infinitesimally rigid Hermitian-YangMills (HYM) connections on $\mathscr{R}_{\rho}$.

In this paper we consider the case $n=3$ and $G$ a finite subgroup of $\operatorname{SL}(3, \mathbb{C})$ acting freely on $\mathbb{C}^{3} \backslash\{0\}$; this condition is equivalent, in retrospect, with the existence of ALE crepant resolutions $\pi: X \rightarrow \mathbb{C}^{3} / G$. From the classification of finite subgroups of $\mathrm{SL}(3, \mathbb{C})$ started by Blichfeldt [6] and completed by Yau and $\mathrm{Yu}$ [29], $G$ must be abelian ${ }^{1}$. From the work of Bridgeland, King, and Reid [7] and Craw and Ishii [10], it is known that all projective crepant resolutions of such $\mathbb{C}^{3} / G$ can be constructed explicitly via GIT, as moduli spaces $\mathscr{M}_{\theta}$ of $G$-constellations with respect to a generic rational stability parameter $\theta$. Moreover, they are naturally equipped with a collection of tautological line bundles $\left\{\mathscr{R}_{\rho}: \rho \in \operatorname{Irr}(G)\right\}$. Because of the relation between GIT

\footnotetext{
${ }^{1}$ Such a group must also be isomorphic to $\mathbb{Z}_{n}$ with $n$ odd, see Remark 3.8. However, for this work, it is only relevant that $G$ is abelian and that it acts freely on $\mathbb{C}^{3} \backslash\{0\}$.
} 
and Kähler reduction, the analytification ${ }^{2} M_{\theta}:=\mathscr{M}_{\theta}^{\text {an }}$ carries a canonical Kähler metric and $\mathcal{R}_{\rho}:=\mathscr{R}_{\rho}^{\text {an }}$ carries a natural Hermitian connection. In the two dimensional situation considered by Kronheimer and Nakajima, the hyperkähler condition ensures automatically that the metric on $M_{\theta}$ is Ricci-flat and the connection on $\mathcal{R}_{\rho}$ is HYM. In dimension three this is no longer true. The main result of this paper shows that they can be deformed to satisfy these conditions and, most importantly, the resulting connection on $\mathcal{R}=\bigoplus_{\rho} \mathcal{R}_{\rho}$ is infinitesimally rigid.

Theorem 1.1. Let $G$ be a finite subgroup of $\mathrm{SL}(3, \mathbb{C})$ acting freely on $\mathbb{C}^{3} \backslash\{0\}$ and let $\theta \in \Theta_{\mathbb{Q}}$ be a generic rational stability parameter. Then the following hold:

(1) $M_{\theta}$ carries a Ricci-flat ALE Kähler metric $g_{\theta, \mathrm{RF}}$.

(2) For each $\rho \in \operatorname{Irr}(G)$ the tautological holomorphic line bundle $\mathcal{R}_{\rho}$ carries an asymptotically flat HYM connection compatible with the holomorphic structure.

(3) The induced HYM connection on the tautological bundle $\mathcal{R}=\bigoplus_{\rho \in \operatorname{Irr}(G)} \mathcal{R}_{\rho}$ is infinitesimally rigid.

Remark 1.2. If $G$ does not act freely on $\mathbb{C}^{3} \backslash\{0\}$, then there are still crepant resolutions $\pi: \mathscr{M}_{\theta} \rightarrow \mathbb{C}^{3} / G$; however, the asymptotic geometry of $M_{\theta}:=\mathscr{M}_{\theta}^{\text {an }}$ will be quasi-asymptotically locally Euclidean (QALE). This causes a large number of additional difficulties. Some of these issues are tackled in recent work of the first named author [12].

Remark 1.3. Theorem 1.1 is of interest in the context of higher dimensional gauge theory, and can be used, for example, to extend the second named author's construction of $\mathrm{G}_{2}$-instantons on generalised Kummer constructions [28] to $\mathrm{G}_{2}$-manifolds arising from $\mathrm{G}_{2}$-orbifolds with codimension 6 singularities.

The existence of the Ricci-flat Kähler metric on $M_{\theta}$ is a consequence of Joyce's proof of the Calabi conjecture for ALE crepant resolutions [19, Section 8], while the existence of the HYM connection is a simple consequence of the properties of the Laplace operator on ALE manifolds. The most interesting and difficult part of Theorem 1.1 is the infinitesimal rigidity statement. This will be a consequence of a vanishing result in Lemma 5.1, whose proof relies heavily on properties of the bounded derived category of coherent sheaves on $\mathscr{M}_{\theta}$ and consequences of the derived category McKay correspondence. This is in stark contrast with the work of Kronheimer and Nakajima [22] who prove infinitesimal rigidity by bare hands.

By a result of Craw and Ishii, see Theorem 2.3, the tautological line bundles $\left\{\mathcal{R}_{\rho}: \rho \in \operatorname{Irr}(G)\right\}$ form a basis in the $K$-theory of $M_{\theta}$ and thus their Chern characters form a basis of $H^{*}\left(M_{\theta}, \mathbb{R}\right)$. Since in our set-up $G$ acts freely on $\mathbb{C}^{3} \backslash\{0\}$, the exceptional divisor is contained in a compact subset of $M_{\theta}$ whose complement is homeomorphic to a truncated cone over $S^{5} / G$ and therefore $H^{2}\left(M_{\theta}, \mathbb{R}\right) \cong H_{c}^{2}\left(M_{\theta}, \mathbb{R}\right)$. Hence we have the triple product

$$
\int_{M_{\theta}}: H^{2}\left(M_{\theta}, \mathbb{R}\right)^{\otimes 3} \cong H_{c}^{2}\left(M_{\theta}, \mathbb{R}\right)^{\otimes 3} \rightarrow \mathbb{R} .
$$

Exploiting the infinitesimal rigidity and using the Atiyah-Patodi-Singer index theorem applied to carefully chosen Dirac operators we derive the following result for this triple product.

Theorem 1.4. Let $G$ be a finite subgroup of $\operatorname{SL}(3, \mathbb{C})$ acting freely on $\mathbb{C}^{3} \backslash\{0\}$. Then for every generic rational stability parameter $\theta \in \Theta_{\mathbb{Q}}$, we have

$$
\frac{1}{2} \int_{M_{\theta}} c_{1}\left(\mathcal{R}_{\rho}\right)^{2} c_{1}\left(\mathcal{R}_{\sigma}\right)-c_{1}\left(\mathcal{R}_{\rho}\right) c_{1}\left(\mathcal{R}_{\sigma}\right)^{2}=-\left(C^{-1}\right)_{\rho \sigma}
$$

for all $\rho, \sigma \in \operatorname{Irr}_{0}(G)$. Here $C$ is a matrix which depends only on $G \subset \mathrm{SL}(3, \mathbb{C})$, see (7.4).

\footnotetext{
${ }^{2}$ For more details on analytification, the passage from the algebraic to the analytic category, we recommend Neeman's book [24].
} 
Remark 1.5. If we denote by $\widetilde{c h}:=\mathrm{ch}-\mathrm{rk}$ the reduced Chern character, then (1.2) can equivalently be written as

$$
\int_{M_{\theta}} \tilde{\operatorname{ch}}\left(\mathcal{R}_{\rho}\right) \widetilde{\operatorname{ch}}\left(\mathcal{R}_{\sigma}^{*}\right)=-\left(C^{-1}\right)_{\rho \sigma}
$$

which is the natural analogue of Kronheimer and Nakajima's [22, Theorem A.7] giving their geometrical interpretation of the McKay correspondence.

It has been pointed out to us by a referee of an earlier version of this paper, that the formula (1.2) could also be derived from the work of Ito and Nakajima [18, Corollary 5.3] by applying the Riemann-Roch theorem for quasi-projective varieties [5].

Note that Theorem 1.4 exhibits a certain part of this triple product (1.1) that does not depend on the specific choice of crepant resolution but only on the subgroup $G$ of $\operatorname{SL}(3, \mathbb{C})$. It is now interesting to ask precisely how much of (1.1) is independent of the choice of crepant resolution and to try to determine (1.1) depending on $\theta$. To our knowledge both of these question are still open and deserve to be investigated in future work.

The paper is organised as follows: In Section 2 we briefly recall the construction of crepant resolutions as moduli spaces of $G$-constellations, introduce the Fourier-Mukai transform and collect the results of Bridgeland, King and Reid [7] and Craw and Ishii [10] that are relevant for our work. In Section 3 we present the construction of moduli spaces of $G$-constellations from the Kähler point of view and discuss their geometry in more detail. In Section 4 we prove the first two parts of Theorem 1.1, while in Section 5 we prove the infinitesimal rigidity statement. Section 6 introduces the Dirac operators on $M_{\theta}$ relevant for the proof of Theorem 1.4, establishes their main properties, and uses the build-up of work so far to prove the vanishing of the index in Proposition 6.2. We complete the proof of Theorem 1.4 in Section 7.

\section{Moduli spaces of $G$-constellations}

Let $G$ be a finite subgroup of $\operatorname{SL}(3, \mathbb{C})$. We denote by $\operatorname{Irr}(G)$ its set of irreducible representations, by $\operatorname{Rep}(G)$ its representation ring, and by $R$ its regular representation. Moreover, $G$ has a natural action on $\mathbb{C}^{3}$ which we tacitly assume throughout this article.

Definition 2.1. A $G$-sheaf on $\mathbb{C}^{3}$ is a coherent sheaf $\mathscr{F}$ together with an action of $G$ which is equivariant with respect to the action of $G$ on $\mathbb{C}^{3}$. A $G$-sheaf is called a $G$-constellation if $H^{0}\left(\mathbb{C}^{3}, \mathscr{F}\right) \cong R$ as $G$-modules. Two $G$-constellations are isomorphic if they are isomorphic as $G$-sheaves.

From this definition it follows that the set-theoretic support of a $G$-constellation is a finite union of $G$-orbits. Thus a $G$-constellation is a sheaf-theoretic generalisation of the notion of G-orbit.

Definition 2.2. The set

$$
\Theta:=\left\{\theta \in \operatorname{Hom}_{\mathbb{Z}}(\operatorname{Rep}(G), \mathbb{Z}): \theta(R)=0\right\}
$$

is called the space of integral stability parameters. The sets $\Theta_{\mathbb{Q}}:=\Theta \otimes_{\mathbb{Z}} \mathbb{Q}$ and $\Theta_{\mathbb{R}}:=\Theta \otimes_{\mathbb{Z}} \mathbb{R}$ are called the space of rational stability parameters and the space of real stability parameters, respectively. Given $\theta \in \Theta_{\mathbb{R}}$, a $G$-constellation $\mathscr{F}$ is called $\theta$-stable (resp. $\theta$-semi-stable) if each non-trivial proper $G$-equivariant subsheaf $\mathscr{E} \subset \mathscr{F}$ satisfies $\theta\left(H^{0}\left(\mathbb{C}^{3}, \mathscr{E}\right)\right)>0$ (resp. $\left.\theta\left(H^{0}\left(\mathbb{C}^{3}, \mathscr{E}\right)\right) \geq 0\right)$.

When $\theta$ is a rational stability parameter GIT techniques are used to prove that the $\theta$-stable $G$-constellations form a fine moduli space $\mathscr{M}_{\theta}$. 
Theorem 2.3 (Craw and Ishii [10, Section 2.1]). If $\theta \in \Theta_{\mathbb{Q}}$, then there exists a fine moduli space $\mathscr{M}_{\theta}$ of $\theta$-stable $G$-constellations on $\mathbb{C}^{3}$. Moreover, for each representation $\rho$ of $G$ there exists a locally free sheaf $\mathscr{R}_{\rho}$ on $\mathscr{M}_{\theta}$. If $\rho$ and $\sigma$ are two representations of $G$, then $\mathscr{R}_{\rho \oplus \sigma}=\mathscr{R}_{\rho} \oplus \mathscr{R}_{\sigma}$.

Sketch of the proof. The construction of $\mathscr{M}_{\theta}$ is based on ideas of King [20] and Sardo Infirri [25]. A $G$-constellation on $\mathbb{C}^{3}$ is a $G$-equivariant $\operatorname{Sym}^{\bullet}\left(\mathbb{C}^{3}\right)^{*}$-module structure on $R$, i.e., a $G$-equivariant homomorphism $\operatorname{Sym}^{\bullet}\left(\mathbb{C}^{3}\right)^{*} \rightarrow \operatorname{End}(R)$. Hence, each point $B$ in

$$
N:=\left\{B \in\left(\operatorname{End}(R) \otimes \mathbb{C}^{3}\right)^{G}:[B \wedge B]=0 \in \operatorname{End}(R) \otimes \Lambda^{2} \mathbb{C}^{3}\right\}
$$

defines a $G$-constellation via $p \in \operatorname{Sym}^{\bullet}\left(\mathbb{C}^{3}\right)^{*} \mapsto p(B) \in \operatorname{End}(R)$. (Here $[\cdot \wedge \cdot]$ is composed of the commutator $[\cdot, \cdot]: \operatorname{End}(R) \otimes \operatorname{End}(R) \rightarrow \operatorname{End}(R)$ and the wedge product $\wedge: \mathbb{C}^{3} \otimes \mathbb{C}^{3} \rightarrow \Lambda^{2} \mathbb{C}^{3}$.) In fact, every $G$-constellation arises in this way. Two points in $N$ yield isomorphic $G$-constellations if and only if they are related by a $G$-equivariant automorphism of $R$, i.e., an element of $\operatorname{GL}(R)^{G}$. Since $R=\bigoplus_{\rho \in \operatorname{Irr}(G)} \mathbb{C}^{\operatorname{dim} \rho} \otimes \rho$, Schur's lemma gives

$$
\mathrm{GL}(R)^{G}=\prod_{\rho \in \operatorname{Irr}(G)} \mathrm{GL}\left(\mathbb{C}^{\operatorname{dim} \rho}\right)
$$

Because the diagonal $\mathbb{C}^{*} \subset \mathrm{GL}(R)^{G}$ acts trivially on $N$, the action of $\operatorname{GL}(R)^{G}$ descends to an action of $\operatorname{PGL}(R)^{G}$.

An integral stability parameter $\theta \in \Theta$ determines a character $\chi_{\theta}: \mathbb{P G L}(R)^{G} \rightarrow \mathbb{C}^{*}$ defined by

$$
\chi_{\theta}([g])=\chi_{\theta}\left(\left[\left(g_{\rho}\right)\right]\right):=\prod_{\rho \in \operatorname{Irr}(G)} \operatorname{det}\left(g_{\rho}\right)^{\theta(\rho)} .
$$

King [20, Proposition 3.1] proved that an element of $N$ is stable (resp. semi-stable) in the sense of GIT with respect to $\chi_{\theta}$ if and only if the corresponding $G$-constellation is $\theta$-stable (resp. $\theta$-semi-stable). Let $N_{\theta}^{s}$ (resp. $N_{\theta}^{s s}$ ) be the subset of GIT (semi-)stable points with respect to $\chi_{\theta}$ in $N$ and let

$$
\mathscr{M}_{\theta}:=N_{\theta}^{s} / \mathbb{P G L}(R)^{G}
$$

be the corresponding GIT quotient. As schemes, $\mathscr{M}_{k \theta}=\mathscr{M}_{\theta}$ for any $k \in \mathbb{N}$; therefore, the above construction extends to rational stability parameters $\theta \in \Theta_{\mathbb{Q}}$ as well ${ }^{3}$.

To see that $\mathscr{M}_{\theta}$ is indeed a fine moduli space of $\theta$-stable $G$-constellations, we construct a universal $G$-constellation $\mathscr{U}_{\theta}$ on $\mathscr{M}_{\theta} \times \mathbb{C}^{3}$. For this purpose we identify

$$
\operatorname{PGL}(R)^{G} \cong \prod_{\rho \in \operatorname{Irr}_{0}(G)} \mathrm{GL}\left(\mathbb{C}^{\operatorname{dim} \rho}\right)
$$

where $\operatorname{Irr}_{0}(G)$ is the set of non-trivial irreducible representations of $G$. In this way $\mathbb{P G L}(R)^{G}$ acts on $R$. This makes $R \otimes \mathscr{O}_{N}$ into a $\mathbb{P G L}(R)^{G}$-equivariant sheaf on $N$. We denote its descend to $\mathscr{M}_{\theta}$ by $\mathscr{R}$. Since the universal morphism $R \otimes \mathscr{O}_{N} \rightarrow \mathbb{C}^{3} \otimes R \otimes \mathscr{O}_{N}$ is $\mathbb{P G L}(R)^{G}$-equivariant, it descends to a universal morphism $\mathscr{R} \rightarrow \mathbb{C}^{3} \otimes \mathscr{R}$ on $\mathscr{M}_{\theta}$. This determines the universal $G$-constellation $\mathscr{U}_{\theta}$ on $\mathscr{M}_{\theta} \times \mathbb{C}^{3}$. Concretely, $\mathscr{U}_{\theta}$ is the sheaf obtained by pulling back $\mathscr{R}$ to $\mathscr{M}_{\theta} \times \mathbb{C}^{3}$ (via the projection to $\mathscr{M}_{\theta}$ ) with the action of $\mathscr{O}_{\mathbb{C}^{3}}=\operatorname{Sym} \bullet\left(\mathbb{C}^{3}\right)^{*}$ prescribed by the universal morphism.

Let $\rho: G \rightarrow \operatorname{Aut}\left(R_{\rho}\right)$ be a representation of $G$. Then $\mathbb{P G L}(R)^{G}$ acts on $R_{\rho}$ via the identification (2.3) and as above we can associate with $\rho$ a locally free sheaf $\mathscr{R}_{\rho}$ on $\mathscr{M}_{\theta}$. It is clear from the construction that $\mathscr{R}_{\rho \oplus \sigma}=\mathscr{R}_{\rho} \oplus \mathscr{R}_{\sigma}$.

\footnotetext{
${ }^{3}$ For each $\theta \in \Theta_{\mathbb{Q}}$, we can find $k \in \mathbb{N}$ so that $k \theta \in \Theta$. We set $\mathscr{M}_{\theta}:=\mathscr{M}_{k \theta}$. By what was said earlier, this does only depends on $\theta$.
} 
Remark 2.4. When a $G$-constellation is $\theta$-stable for some real stability parameter $\theta \in \Theta_{\mathbb{R}}$, its set-theoretic support is a unique $G$-orbit of $\mathbb{C}^{3}$. Thinking of this as a point in $\mathbb{C}^{3} / G$, we obtain a well-defined map $\pi_{\theta}: \mathscr{M}_{\theta} \rightarrow \mathbb{C}^{3} / G$.

To obtain further insight into the spaces $\mathscr{M}_{\theta}$ and the properties of the map $\pi_{\theta}$, it is helpful to use the language of derived categories. We first recall the bounded derived category $D(\mathscr{A})$ associated with an abelian category $\mathscr{A}$. For details we refer the reader to Bühler's notes [8] as well as Thomas' article [26] and Huybrechts' book [17], both of which underline the importance of derived categories of coherent sheaves in algebraic geometry. Roughly speaking, $D(\mathscr{A})$ is obtained from the category of bounded chain complexes in $\mathscr{A}$ by formally inverting quasiisomorphisms. If $A, B \in \mathscr{A}$ are considered as bounded chain complexes concentrated in degree zero, then $\operatorname{Hom}_{D(\mathscr{A})}(A, B)$ is a complex whose cohomology computes $\operatorname{Ext}^{\bullet}(A, B)$, that is,

$$
H^{\bullet}\left(\operatorname{Hom}_{D(\mathscr{A})}(A, B)\right)=\operatorname{Ext}^{\bullet}(A, B) .
$$

If $\mathscr{B}$ is another abelian category and $f: \mathscr{A} \rightarrow \mathscr{B}$ is a left-exact functor, then one assigns to it a right derived functor $\mathbf{R} f: D(\mathscr{A}) \rightarrow D(\mathscr{B})$. If $A \in \mathscr{A}$ is considered as a bounded chain complex concentrated in degree zero, then $\mathbf{R} f(A)$ is a complex which computes $R^{\bullet} f(A)$, that is,

$$
H^{\bullet}(\mathbf{R} f(A))=R^{\bullet} f(A) .
$$

An analogous construction assigns to every right-exact functor $g: \mathscr{A} \rightarrow \mathscr{B}$ a left derived functor $\mathbf{L} g$. As is customary when working with derived categories, we will often write $f$ and $g$ instead of $\mathbf{R} f$ and $\mathbf{L} g$.

An important example of a derived category is $D(\operatorname{Coh}(X))$, the bounded derived category of coherent sheaves over a scheme $X$. If $X$ and $Y$ are two schemes and $K \in \operatorname{Coh}(X \times Y)$ is a coherent sheaf, then the Fourier-Mukai transform with kernel $K$ is the functor $\Phi_{K}: D(\operatorname{Coh}(X)) \rightarrow$ $D(\operatorname{Coh}(Y))$ defined by

$$
\Phi_{K}(-):=\left(p_{2}\right)_{*}\left(p_{1}^{*}-\otimes K\right) .
$$

Here $p_{1}^{*},\left(p_{2}\right)_{*}$ and $\otimes$ are taken in the derived sense, with $p_{1}$ and $p_{2}$ denoting the projections from $X \times Y$ to $X$ and $Y$ respectively. A simple instance of a Fourier-Mukai transform is the following: If $f: X \rightarrow Y$ is a morphism and $\mathscr{O}_{\Gamma}$ denotes the structure sheaf of its graph $\Gamma \subset X \times Y$, then $\Phi_{\mathscr{O}_{\Gamma}}$ is nothing but $f_{*}$.

In our context, we denote by $D\left(\mathscr{M}_{\theta}\right)$ the bounded derived category of coherent sheaves on $\mathscr{M}_{\theta}$ and by $D^{G}\left(\mathbb{C}^{3}\right)$ the bounded derived category of $G$-sheaves on $\mathbb{C}^{3}$, which is the same as the bounded derived category $D\left(\left[\mathbb{C}^{3} / G\right]\right)$ of coherent sheaves on the stack $\left[\mathbb{C}^{3} / G\right]$. One of the key ideas of Bridgeland, King and Reid [7] was to introduce the Fourier-Mukai transform $\Phi_{\theta}: D\left(\mathscr{M}_{\theta}\right) \rightarrow D^{G}\left(\mathbb{C}^{3}\right)$ whose kernel is given by the universal $G$-constellation $\mathscr{U}_{\theta}$

$$
\Phi_{\theta}(-)=q_{*}\left(p^{*}\left(-\otimes \rho_{0}\right) \otimes \mathscr{U}_{\theta}\right)
$$

to the study of crepant resolutions. Here $p: \mathscr{M}_{\theta} \times \mathbb{C}^{3} \rightarrow \mathscr{M}_{\theta}$ and $q: \mathscr{M}_{\theta} \times \mathbb{C}^{3} \rightarrow \mathbb{C}^{3}$ are the canonical projections and $\rho_{0}$ is the trivial representation of $G$.

Definition 2.5. A real stability parameter $\theta \in \Theta_{\mathbb{R}}$ is called generic, if there exists no non-trivial proper subrepresentation $S \subset R$ such that $\theta(S)=0$.

The space of generic stability parameters is dense in $\Theta_{\mathbb{R}}$. Moreover, if $\theta \in \Theta_{\mathbb{R}}$ is generic, then every $\theta$-semi-stable $G$-constellation is $\theta$-stable (see also [10, Section 2.2]). In the particular case when $\theta$ is a generic rational stability parameter, the above techniques are used to prove that $\mathscr{M}_{\theta}$ together with the map $\pi_{\theta}$ defined in Remark 2.4 is a crepant resolution of singularities of $\mathbb{C}^{3} / G$. 
Theorem 2.6 (Craw and Ishii [10, Proposition 2.2 and Theorem 2.5]). For each $\theta \in \Theta_{\mathbb{Q}}$ generic, the morphism $\pi_{\theta}: \mathscr{M}_{\theta} \rightarrow \mathbb{C}^{3} / G$ is a projective crepant resolution and the Fourier-Mukai transform $\Phi_{\theta}$ is an equivalence of derived categories. Moreover, the locally free sheaves $\mathscr{R}_{\rho}$ form $a \mathbb{Z}$-basis in $K$-theory.

Remark 2.7. Bridgeland, King and Reid [7] first proved this result for Nakamura's G-Hilbert scheme. Craw and Ishii observed that their proof works more generally for moduli spaces of $G$-constellations. In the course of the proof of the fact that $\Phi_{\theta}$ is an equivalence of derived categories one has to show that $\mathscr{M}_{\theta}$ is smooth. This is achieved by appealing to a deep result from commutative algebra called the intersection theorem [7, Theorem 7.1]. That $\pi_{\theta}$ is a crepant resolution then follows from a categorical criterion for a resolution to be crepant [7, Lemma 3.1].

Furthermore, for abelian subgroups $G$ of $\mathrm{SL}(3, \mathbb{C})$ toric geometry techniques are used to prove a partial converse of Theorem 2.6.

Theorem 2.8 (Craw and Ishii [10, Theorem 1.1]). If $G$ is an abelian subgroup of SL(3, $\mathbb{C})$, then every projective crepant resolution of $\mathbb{C}^{3} / G$ is a moduli space of $\theta$-stable $G$-constellations for some generic $\theta \in \Theta_{\mathbb{Q}}$.

\section{$3 \quad \mathscr{M}_{\theta}$ via Kähler reduction}

We now approach the previous discussion from the Kähler point of view. There is no loss in assuming that the finite group $G \subset \mathrm{SL}(3, \mathbb{C})$ preserves the standard Hermitian metric on $\mathbb{C}^{3}$, that is, $G \subset \mathrm{SU}(3)$. We also fix a $G$-invariant Hermitian metric on $R$. Then the vector space $\left(\operatorname{End}(R) \otimes \mathbb{C}^{3}\right)^{G}$ naturally is a Kähler manifold with Kähler form

$$
\omega(B, C):=\operatorname{Im} \sum_{\alpha=1}^{3} \operatorname{tr}\left(B_{\alpha} C_{\alpha}^{*}\right)=\sum_{\alpha=1}^{3} \frac{1}{2 i} \operatorname{tr}\left(B_{\alpha} C_{\alpha}^{*}-B_{\alpha}^{*} C_{\alpha}\right) .
$$

Here we identify $B \in\left(\operatorname{End}(R) \otimes \mathbb{C}^{3}\right)^{G}$ with a triple $\left(B_{1}, B_{2}, B_{3}\right)$ of endomorphisms of $R$.

Proposition 3.1. The action of $\mathbb{P U}(R)^{G}$ on $\left(\operatorname{End}(R) \otimes \mathbb{C}^{3}\right)^{G}$ by conjugation is Hamiltonian with moment map $\mu:\left(\operatorname{End}(R) \otimes \mathbb{C}^{3}\right)^{G} \rightarrow\left(\mathfrak{p u}(R)^{G}\right)^{*}$ given by

$$
\langle\mu(B), \xi\rangle=\sum_{\alpha} \frac{1}{2 i} \operatorname{tr}\left(\xi\left[B_{\alpha}, B_{\alpha}^{*}\right]\right)
$$

Proof. It is enough to prove this for the action of $U(R)^{G}$. If $\xi \in \mathfrak{u}(R)^{G}$, then the corresponding vector field $X_{\xi}$ on $\left(\operatorname{End}(R) \otimes \mathbb{C}^{3}\right)^{G}$ is given by $X_{\xi}(B)=[\xi, B]$. Thus

$$
\begin{aligned}
i\left(X_{\xi}\right) \omega(\hat{B}) & =\sum_{\alpha} \frac{1}{2 i} \operatorname{tr}\left(\left[\xi, B_{\alpha}\right] \hat{B}_{\alpha}^{*}-\left[\xi, B_{\alpha}\right]^{*} \hat{B}_{\alpha}\right) \\
& =\sum_{\alpha} \frac{1}{2 i} \operatorname{tr}\left(\xi\left(\left[B_{\alpha}, \hat{B}_{\alpha}^{*}\right]+\left[\hat{B}_{\alpha}, B_{\alpha}^{*}\right]\right)\right)=\langle\mathrm{d} \mu(B) \hat{B}, \xi\rangle .
\end{aligned}
$$

To continue, we first need to analyze the relation between $\left(\mathfrak{p u}(R)^{G}\right)^{*}$ and the spaces of stability parameters introduced in Definition 2.2. For each $\theta \in \Theta_{\mathbb{R}}$, we define $\zeta_{\theta} \in\left(\mathfrak{p u}(R)^{G}\right)^{*}$ by

$$
\zeta_{\theta}(\xi):=-\sum_{\rho \in \operatorname{Irr}(G)} i \theta(\rho) \operatorname{tr}\left(\xi \cdot \pi_{\rho}\right)
$$


for all $\xi \in \mathfrak{p u}(R)^{G}$. Here $\pi_{\rho}: R \rightarrow \mathbb{C}^{\operatorname{dim} \rho} \otimes R_{\rho}$ is the projection onto the $\rho$-isotypical component of the regular representation and $\xi \cdot \pi_{\rho}$ is thought of as an element in $\operatorname{End}(R)$. Since $\left\{i \pi_{\rho} \mid \rho \in\right.$ $\operatorname{Irr}(G)\}$ spans the center of $\mathfrak{u}(R)^{G}$, we can identify $\Theta_{\mathbb{R}}$ with the centre of $\left(\mathfrak{p u}(R)^{G}\right)^{*}$ via $\theta \mapsto \zeta_{\theta}$. Under this identification, the generic stability parameters $\theta \in \Theta_{\mathbb{R}}$ correspond to $\zeta_{\theta}$ satisfying $\zeta_{\theta}\left(i \pi_{S}\right) \neq 0$ for all non-trivial proper subrepresentations $S \subset R$. Here $\pi_{S}: R \rightarrow S$ denotes the orthogonal projection onto $S$. Moreover, when $\theta$ is integral, then $\zeta_{\theta}=-i \mathrm{~d} \chi_{\theta} \in\left(\mathfrak{p u}(R)^{G}\right)^{*}$ with $\chi_{\theta}$ the character defined in (2.2).

With the above identification, for each $\theta \in \Theta_{\mathbb{R}}$ we let

$$
M_{\theta}:=\left(N \cap \mu^{-1}\left(\zeta_{\theta}\right)\right) / \mathbb{P U}(R)^{G}
$$

be the corresponding Kähler quotient of the restriction of the moment map $\mu$ to $N$ defined in (2.1). Note that $N$ is a complex subvariety of $\left(\operatorname{End}(R) \otimes \mathbb{C}^{3}\right)^{G}$ and thus the Kähler quotient makes sense. The space $M_{\theta}$ comes with a natural set of bundles constructed the following way: For each representation $\rho: G \rightarrow \mathrm{GL}\left(R_{\rho}\right)$ of $G$, the group $\mathbb{P U}(R)^{G}$ acts on $R_{\rho}$ via (2.3). Let

$$
\mathcal{R}_{\rho}:=\left(N \cap \mu^{-1}(\theta)\right) \times_{\mathbb{P U}(R)^{G}} R_{\rho}
$$

be the associated complex vector bundle over $M_{\theta}$. As we will show in a moment, the bundles $\mathcal{R}_{\rho}$ carry natural holomorphic structures. We call $\mathcal{R}_{\rho}$ the tautological (holomorphic) bundle associated with $\rho$.

We proceed now to describe the relation between $\mathscr{M}_{\theta}$ and $\mathscr{R}_{\rho}$ defined in Theorem 2.3 and the $M_{\theta}$ and $\mathcal{R}_{\rho}$ defined above. Note that the first makes sense only for the rational stability parameters $\theta$, while the second makes sense for all $\theta \in \Theta_{\mathbb{R}}$.

Proposition 3.2. If $B \in N \cap \mu^{-1}\left(\zeta_{\theta}\right)$, then the $G$-constellation $\mathscr{F}$ associated to $B$ is $\theta$-semistable. Therefore we have $N \cap \mu^{-1}\left(\zeta_{\theta}\right) \subset N_{\theta}^{s s}$ for all $\theta \in \Theta_{\mathbb{R}}$.

Proof. Let $\mathscr{E}$ be a non-trivial proper $G$-equivariant subsheaf of $\mathscr{F}$. Then the regular representation decomposes into two non-trivial proper subrepresentations $R=S \oplus T$ with $S:=H^{0}\left(\mathbb{C}^{3}, \mathscr{E}\right)$ and $T$ its orthogonal complement. Corresponding to $\mathscr{E}$ there is an associated triple of matrices $C \in \operatorname{End}(S) \otimes \mathbb{C}^{3}$. Moreover, since each component of $B$ leaves $S$ invariant, $B=\left(\begin{array}{ll}C & D \\ 0 & E\end{array}\right)$ with $D \in \operatorname{Hom}(T, S) \otimes \mathbb{C}^{3}$ and $E \in \operatorname{End}(S) \otimes \mathbb{C}^{3}$. Therefore,

$$
\left\langle\mu(B), i \pi_{S}\right\rangle=\frac{1}{2} \operatorname{tr}_{S}\left(\left[C, C^{*}\right]+D D^{*}\right)=\frac{1}{2} \operatorname{tr}_{S}\left(D D^{*}\right) \geq 0 .
$$

Since $\left\langle\mu(B), i \pi_{S}\right\rangle=\zeta_{\theta}\left(i \pi_{S}\right)=\theta(S)$, it follows that $\theta\left(H^{0}\left(\mathbb{C}^{3}, \mathscr{E}\right)\right) \geq 0$.

King [20, Theorem 6.1] proved the following version of the Kempf-Ness theorem: If $\theta \in \Theta$, then each closed $\mathbb{P G L}(R)^{G}$-orbit in $N_{\theta}^{s s}$ meets $N \cap \mu^{-1}\left(\zeta_{\theta}\right)$ in precisely one $\mathbb{P U}(R)^{G}$-orbit. From this we obtain the following identification:

Proposition 3.3. Suppose that $\theta \in \Theta_{\mathbb{Q}}$ is generic. Then the inclusion $N \cap \mu^{-1}\left(\zeta_{\theta}\right) \hookrightarrow N_{\theta}^{s}=N_{\theta}^{s s}$ induces a biholomorphic map from $M_{\theta}$ to the analytification of $\mathscr{M}_{\theta}$. This map identifies the complex vector bundle $\mathcal{R}_{\rho}$ with the complex vector bundle underlying the analytification of the locally free sheaf $\mathscr{R}_{\rho}$.

The identification with $\mathscr{R}_{\rho}^{\text {an }}$ equips $\mathcal{R}_{\rho}$ with a holomorphic structure. Moreover, by Theorem 2.3 we have

$$
\mathcal{R}_{\rho \oplus \sigma}=\mathcal{R}_{\rho} \oplus \mathcal{R}_{\sigma}
$$

as holomorphic vector bundles. 
The Kähler quotient constrution above induces a metric $g_{\theta}$ and a Kähler form $\omega_{\theta}$ on $M_{\theta}$ for each $\theta \in \Theta_{\mathbb{R}}$. We also have a canonical connection $A_{\theta}$ on the $\mathbb{P U}(R)^{G}$-bundle $\mu^{-1}\left(\zeta_{\theta}\right) \rightarrow M_{\theta}$ whose horizontal space at $B \in \mu^{-1}\left(\zeta_{\theta}\right)$ is given by the orthogonal complement in $T_{B} \mu^{-1}\left(\zeta_{\theta}\right)$ of the tangent space to $\mathbb{P U}(R)^{G}$-orbit through $B$. In the case when $G$ is a finite subgroup of $\mathrm{SU}(3)$ which acts freely on $\mathbb{C}^{3} \backslash\{0\}$ a more precise description of the geometry of $M_{\theta}$ and $\mathcal{R}_{\rho}$ can be given. For this, we first need to recall a number of definitions.

Definition 3.4. Let $G$ be a finite subgroup of $\mathrm{SO}(n)$ acting freely on $\mathbb{R}^{n} \backslash\{0\}$. A Riemannian manifold $(X, g)$ is called an asymptotically locally Euclidean (ALE) manifold asymptotic to $\mathbb{R}^{n} / G$ to order $\tau>0$ if there exists a compact subset $K \subset X$ and a diffeomorphism $\pi:\left(\mathbb{R}^{n} \backslash \bar{B}_{1}\right) / G \rightarrow$ $X \backslash K$ so that

$$
\left|\partial_{k}\left(\pi^{*} g-g_{0}\right)\right|_{g_{0}}=O\left(r^{-\tau-k}\right)
$$

for all $0 \leq k \leq 2$. Here we use the notation $r:=|x|$. In the above situation we also say that the metric $g$ is asymptotically locally Euclidean (ALE) of order $\tau$.

Definition 3.5. Let $H$ be a Lie group. A connection $A$ on a $H$-bundle $E$ over an ALE manifold $(X, g)$ asymptotic to $\mathbb{R}^{n} / G$ is called asymptotically flat of order $\tau>0$ if there exists a flat connection $A_{0}$ on $\left.E\right|_{X \backslash K}$ such that

$$
\left|\nabla_{A_{0}}^{k}\left(A-A_{0}\right)\right|=O\left(r^{-\tau-k}\right)
$$

for $0 \leq k \leq 1$. Here we use a metric which is induced by the Euclidean metric on $\mathbb{R}^{n}$ and a metric on the adjoint bundle associated with $E$ which is parallel with respect to $A_{0}$.

With these definitions we can now characterise the geometry of $M_{\theta}$ and of the corresponding tautological bundles $\mathcal{R}_{\rho}$.

Theorem 3.6. Let $G$ be a finite subgroup of $\mathrm{SU}(3)$ acting freely on $\mathbb{C}^{3} \backslash\{0\}$. Then the following hold:

(1) $\left(M_{0}, g_{0}\right)$ is isometric to the orbifold $\mathbb{C}^{3} / G$ with the induced orbifold Kähler metric. The corresponding connection $A_{0}$ is flat.

(2) If $\theta \in \Theta_{\mathbb{Q}}$ is generic, then $M_{\theta}$ is smooth and the induced Kähler metric $g_{\theta}$ is ALE of order 4 .

(3) If $\theta \in \Theta_{\mathbb{Q}}$ generic, then the $\mathbb{P U}(R)^{G}$-connection $A_{\theta}$ is asymptotically flat of order 1 . Its curvature decays like $r^{-4}$ and is of type $(1,1)$. Moreover, the induced connection $A_{\theta, \rho}$ on $\mathcal{R}_{\rho}$ is compatible with the unique holomorphic structure on $\mathcal{R}_{\rho}$ for each $\rho \in \operatorname{Irr}(G)$.

In the case of finite subgroups of $\mathrm{SU}(2)$ the analogous theorem was proven by Kronheimer [21], Kronheimer and Nakajima [22], and Gocho and Nakajima [13]. For the above theorem, the smoothness of the Kähler quotient $M_{\theta}$ for generic $\theta \in \Theta_{\mathbb{Q}}$ follows from the identification with the algebraic quotient $\mathscr{M}_{\theta}$ provided by Proposition 3.3 and the result of Theorem 2.6. The first statement and the remaining part of the second were proved by Sardo Infirri [25] by generalising Kronheimer's proof. The proof of the first two parts of the third statement is a direct generalisation of the proof in [22, Proposition 2.2] and of Gocho and Nakajima's argument. For the third part, note that the condition that $G$ is a finite subgroup of $\mathrm{SU}(3)$ which acts freely on $\mathbb{C}^{3} \backslash\{0\}$ implies that $G$ is an abelian subgroup, and in fact it must be cyclic of prime order. As a consequence, its irreducible representations are one dimensional, and the corresponding bundles $\mathcal{R}_{\rho}$ are holomorphic line bundles. Since $H^{1}\left(M_{\theta}, \mathcal{O}_{M_{\theta}}\right)=H^{0,1}\left(M_{\theta}\right)=0$, the holomorphic structure on $\mathcal{R}_{\rho}$ is unique for all $\rho \in \operatorname{Irr}(G)$. Therefore it must be compatible with the connection induced by $A_{\theta}$ on $\mathcal{R}_{\rho}$. 
Remark 3.7. The above characterization uses the identification in Proposition 3.3 and therefore the smoothness of $M_{\theta}$ can only be inferred for $\theta$ a generic rational stability parameter. It seems reasonable to expect that $M_{\theta}$ is smooth for all generic real stability parameters $\theta \in \Theta_{\mathbb{R}}$. Because of the homogeneity of the moment map, this is certainly true for all $t \theta$ with $\theta \in \Theta_{\mathbb{Q}}$ generic and $t \in(0, \infty)$. One can show that for generic $\theta \in \Theta_{\mathbb{R}}$ the action of $\mathbb{P} \mathrm{U}(R)^{G}$ on $N \cap \mu^{-1}\left(\zeta_{\theta}\right)$ is free. To conclude that $M_{\theta}$ is smooth, however, one still needs to show that $N \cap \mu^{-1}\left(\zeta_{\theta}\right)$ is contained in the smooth locus of $N$.

Remark 3.8. As mentioned in the introduction, the classification of finite subgroups of $\operatorname{SL}(3, \mathbb{C})$ was initiated by Blichfeldt [6]. He, however, missed two groups, which were found much later by $\mathrm{Yau}$ and $\mathrm{Yu}$ [29]. According to the complete classification, there are ten families of finite subgroups of $\mathrm{SL}(3, \mathbb{C})$. The first family consists of abelian groups acting diagonally on $\mathbb{C}^{3}$; all the other families contain only non-abelian groups. Direct examination shows that these nonabelian groups do not act freely on $\mathbb{C}^{3} \backslash\{0\}$. Using the structure of finite abelian groups, it can be easily seen, that $G$ can only act freely on $\mathbb{C}^{3} \backslash\{0\}$ if it is cyclic. Examining this, it follows that if a finite subgroup of $\operatorname{SL}(3, \mathbb{C})$ acts freely on $\mathbb{C}^{3} \backslash\{0\}$ then it must be isomorphic to $\mathbb{Z}_{n}$ with $n$ odd. This statement, however, depends on the embedding on $\mathbb{Z}_{n}$ into $\operatorname{SL}(3, \mathbb{C})$. For example, $\mathbb{Z}_{9}$ can be embedded into $\mathrm{SL}(3, \mathbb{C})$ in at least two ways: as the subgroup generated by the diagonal matrix $\operatorname{diag}\left(\varepsilon, \varepsilon^{4}, \varepsilon^{4}\right)$, or as the subgroup generated by $\operatorname{diag}\left(\varepsilon, \varepsilon^{3}, \varepsilon^{5}\right)$, with $\varepsilon$ a $9^{\text {th }}$ root of unity. In the first instance $\mathbb{Z}_{9}$ acts freely on $\mathbb{C}^{3} \backslash\{0\}$, in the second, it does not.

\section{Ricci-flat metrics on $M_{\theta}$ and HYM connections on $\mathcal{R}_{\rho}$}

The results of Kronheimer [21] and of Gocho and Nakajima [13] for finite subgroups of SU(2) we alluded to above actually establish that the metric $g_{\theta}$ is Ricci-flat and the induced connections on $\mathcal{R}_{\rho}$ are anti-self-dual and, hence, Hermitian-Yang-Mills (HYM). This is because in dimension two $M_{\theta}$ arises via hyperkähler reduction with the subspace $N$ being the zero locus of the complex component of the hyperkähler moment map. In higher dimension this is no longer the case. Consequently, the metric $g_{\theta}$ on $M_{\theta}$ given by Theorem 3.6 is not necessarily Ricci-flat and the connections $A_{\theta, \rho}$ on $\mathcal{R}_{\rho}$ are not necessarily HYM. Indeed, Sardo Infirri [25, Example 7.1] showed that for $G=\mathbb{Z}_{3} \cong\left\langle\operatorname{diag}\left(e^{2 \pi i / 3}, e^{2 \pi i / 3}, e^{2 \pi i / 3}\right)\right\rangle \subset \mathrm{SU}(3)$ and an appropriate choice of a generic stability parameter, the corresponding Kähler quotient is the total space of the line bundle $\mathcal{O}_{\mathbb{P}^{2}}(-3)$ with a metric which has non-vanishing Ricci curvature. In this section we show that $M_{\theta}$ does admit a Ricci-flat Kähler metric and that the tautological line bundles $\mathcal{R}_{\rho}$ carry asymptotically flat HYM connections, thus proving the first two parts of Theorem 1.1.

The existence of the Ricci-flat Kähler metric follows from the following result:

Theorem 4.1 (Joyce [19, Theorem 8.2.3]). Let $G$ be a finite subgroup of $\mathrm{SU}(n)$ acting freely on $\mathbb{C}^{n} \backslash\{0\}$. Let $X$ be a smooth crepant resolution of $\mathbb{C}^{n} / G$ with an ALE Kähler metric $g$ of order $\tau>n$. Then there exists a unique Ricci-flat ALE Kähler metric $g_{\mathrm{RF}}$ in the Kähler class of $g$. The metric $g_{\mathrm{RF}}$ is $A L E$ of order $2 n$.

Remark 4.2. Joyce states this result only for ALE Kähler metrics of order $\tau=2 n$; however, his proof goes through for $\tau>n$. More specifically, we need to modify the metric $g$ in its Kähler class to be flat outside a compact set and then apply Joyce's proof of the Calabi conjecture in ALE set-up. For this modification of the metric, we need the $d d^{c}$-lemma to hold for a certain exact, real $(1,1)$-form which decays like $\rho^{-\tau}$ on the ALE end. In [19, Theorem 8.4.4] this is done via a Stokes' theorem argument. To use this argument and conclude that the boundary integral is zero, one needs that $\tau>n$.

By Theorem 3.6(2) the induced Kähler metric on $M_{\theta}$ is ALE of order 4 and, hence, the above theorem applies. 
Corollary 4.3. Let $\theta \in \Theta_{\mathbb{Q}}$ be generic and let $\left(M_{\theta}, g_{\theta}\right)$ be the corresponding Kähler quotient. Then there exists a Ricci-flat ALE Kähler metric $g_{\theta, \mathrm{RF}}$ of order 6 on $M_{\theta}$ in the same Kähler class as $g_{\theta}$.

Remark 4.4. Note that in dimension $n \geq 4$, Theorem 4.1 does not apply anymore for the ALE Kähler metrics constructed on crepant resolutions of $\mathbb{C}^{n} / G$ via the Kähler reduction (3.1) since the decay $\tau=4$ is now too weak. Another argument is then needed to show the existence of Ricci-flat ALE Kähler metrics on these crepant resolutions.

We now proceed to show the existence of asymptotically flat HYM connections on the tautological line bundle $\mathscr{R}_{\rho}$. Recall that a $(1,1)$-connection on a complex vector bundle over a Kähler manifold is called Hermitian-Yang-Mills $(H Y M)$ if the contraction of its curvature with the Kähler form vanishes identically, i.e., $\Lambda F_{A}=0$. It turns out that it is a little easier to prove the existence result in terms of Hermitian-Yang-Mills (HYM) metrics. These are Hermitian metrics on holomorphic bundles with the property that their Chern connection, the unique metric connection associated to the holomorphic bundle, is HYM.

Definition 4.5. Let $E$ be a complex vector bundle over an ALE manifold $(X, g)$, let $h_{0}$ be a Hermitian metric on $\left.E\right|_{X \backslash K}$ and let $A_{0}$ be a connection on $\left.E\right|_{X \backslash K}$ compatible with $h_{0}$. A Hermitian metric $h$ on $E$ is called asymptotic to $h_{0}$ to order $\tau>0$ if

$$
\left|\nabla_{A_{0}}^{k}\left(h-h_{0}\right)\right|_{h_{0}}=O\left(r^{-\tau-k}\right)
$$

for all $0 \leq k \leq 2$.

Proposition 4.6. Let $X$ be an ALE Kähler manifold and let $\mathcal{L}$ be a holomorphic line bundle over $X$. If $h_{0}$ is a Hermitian metric on $\mathcal{L}$ such that the curvature $F_{h_{0}}$ of the Chern connection on $\mathcal{L}$ compatible with $h_{0}$ satisfies

$$
\Lambda F_{h_{0}}=O\left(r^{-2-\varepsilon}\right)
$$

for some $\varepsilon>0$, then there exists a HYM metric $h$ on $\mathcal{L}$. Moreover, for every $\tau \in(0, \varepsilon)$ this metric is the unique HYM metric asymptotic to $h_{0}$ to order $\tau$.

From the construction of the bundles $\mathcal{R}_{\rho}$ and of the corresponding connections $A_{\theta, \rho}$ in Theorem 3.6(3), we see that $A_{\theta, \rho}$ is the Chern connection of a Hermitian metric on $\mathcal{R}_{\rho}$. Therefore $\mathcal{R}_{\rho}$ with this Hermitian metric satisfies the conditions of the above proposition yielding the desired existence result:

Corollary 4.7. Let $\theta \in \Theta_{\mathbb{Q}}$ be generic. Then for each $\rho \in \operatorname{Irr}(G)$ the tautological line bundle $\mathcal{R}_{\rho}$ on $M_{\theta}$ carries a $H Y M \mathrm{U}(1)$-connection with respect to $g_{\theta, \mathrm{RF}}$ which is asymptotically flat of order $\tau \in(0,2)$.

Remark 4.8. Using some of the results derived in Section 5, one can show that the HYM connection associated with $h$ in Proposition 4.6 is asymptotically flat of order 5 and, hence, the Hermitian metric $h$ is asymptotic to a flat metric to order 4 .

Remark 4.9. Using heat flow methods, Bando [2] proved that every holomorphic bundle $\mathcal{E}$ over an ALE Kähler manifold which admits a Hermitian metric $h_{0}$ with $\left|F_{h_{0}}\right|=O\left(r^{-2-\varepsilon}\right)$ does in fact carry a HYM metric.

The case of line bundles is much simpler than Bando's result and the proof of Proposition 4.6 follows from the fact that the Laplace operator is an isomorphism between certain weighted Sobolev spaces. Concretely, let $(X, g)$ be an ALE manifold asymptotic to $\mathbb{R}^{n} / G$ as defined in Definition 3.4 and let $r: X \rightarrow[1, \infty)$ denote a smooth extension of the radius function from 
$X \backslash K \cong\left(\mathbb{R}^{n} \backslash \bar{B}_{1}\right) / G$ to all of $X$. For a non-negative integer $k$ and a real number $\delta$ we denote by $W_{\delta}^{k, 2}(X)$ the weighted Sobolev space obtained as the completion of $C_{0}^{\infty}(X)$ with respect the norm

$$
\|f\|_{W_{\delta}^{k, 2}}:=\sum_{j=0}^{k}\left\|r^{-\delta-n / 2+j} \nabla^{j} f\right\|_{L^{2}} .
$$

Let $\Delta_{\delta}: W_{\delta}^{k+2,2}(X) \rightarrow W_{\delta-2}^{k, 2}(X)$ denote the corresponding completion of the Laplacian $\Delta$.

Proposition 4.10 (Bartnik [4, Proposition 2.2]). For $\delta \in(-n+2,0)$ the operator $\Delta_{\delta}$ is an isomorphism.

Sketch of the proof. The weighted Laplacian $\Delta_{\delta}: W_{\delta}^{k+2,2}(X) \rightarrow W_{\delta-2}^{k, 2}(X)$ is a Fredholm operator if and only if the weight parameter $\delta$ is not contained in its set of indicial roots at infinity. This is a discrete set of real numbers which does not intersect the interval $(-n+2,0)$, see Bartnik [4, Sections 1 and 2] for details. Moreover, for $\delta<0$ the kernel of $\Delta_{\delta}$ is trivial by the maximum principle. On the other hand, the cokernel of $\Delta_{\delta}$ is isomorphic to the kernel of its formal adjoint $\Delta_{n-2-\delta}$. Therefore, $\Delta_{\delta}$ is an isomorphism for $\delta \in(-n+2,0)$.

Proof of Proposition 4.6. Any Hermitian metric on $\mathcal{L}$ is of the form $h=e^{f} h_{0}$, for some $f \in C^{\infty}(X)$ and $F_{h}=F_{h_{0}}+\bar{\partial} \partial f \in \Omega^{2}(X, i \mathbb{R})$. Therefore,

$$
i \Lambda F_{h}=i \Lambda F_{h_{0}}+\frac{1}{2} \Delta f \text {. }
$$

Since $\Lambda F_{h_{0}} \in L_{-2-\tau}^{2}(X):=W_{-2-\tau}^{0,2}(X)$ for every $\tau \in(0, \varepsilon)$, by Proposition 4.10 , there exists a unique $f \in W_{-\tau}^{2,2}(X)$ such that $\Delta f=-2 i \Lambda F_{h_{0}}$. Moreover, a computation using the explicit form of the Green function on the end of the ALE manifold $X$ gives that $f=O\left(r^{-\tau}\right)$ for all $0<\tau<\varepsilon$, cf. [19, (proof of) Theorem 8.3.5].

\section{$5 \quad$ Rigidity of HYM connections on the holomorphic tautological bundles}

In this section we prove the infinitesimal rigidity statement in Theorem 1.1(3). This will be an immediate consequence of the following lemma, which is the core vanishing result of this paper.

Lemma 5.1. Let $\theta \in \Theta_{\mathbb{Q}}$ be generic and let $M_{\theta}$ be equipped with an ALE Kähler metric $g$. Let $h$ be a Hermitian metric on the holomorphic bundle $\mathcal{R}=\bigoplus_{\rho \in \operatorname{Irr}(G)} \mathcal{R}_{\rho}$ whose associated Chern connection $A$ is asymptotically flat of order $\tau>0$. Then the space

$$
\mathcal{H}_{A}^{1}:=\left\{a \in \Omega^{0,1}\left(M_{\theta}, \mathcal{E} \operatorname{nd}(\mathcal{R})\right): \bar{\partial}_{A} a=\bar{\partial}_{A}^{*} a=0 \text { and } \lim _{r \rightarrow \infty} \sup _{\partial B_{r}}|a|=0\right\}
$$

is trivial.

Note that if the connection $A$ is HYM, then $\mathcal{H}_{A}^{1}$ is its space of infinitesimal deformations. In particular, it follows that the HYM connection on $\mathcal{R}$ induced by then HYM connections on the bundles $\mathcal{R}_{\rho}$ constructed in the second part of Theorem 1.1 is infinitesimally rigid. This thus completes the proof of the third part of Theorem 1.1.

The strategy for proving Lemma 5.1 is as follows: We first reduce to a vanishing result in complex geometry, see Propositions 5.2 and 5.6. Then since $\theta$ is a generic stability parameter, Proposition 3.3 gives that $M_{\theta}$ is the analytification of the moduli space of $\theta$-stable $G$-constellations $\mathscr{M}_{\theta}$. Using GAGA, we translate the vanishing into an algebraic geometry problem, see (5.3), which we then solve using the results of Bridgeland, King and Reid [7] and Craw 
and Ishii [10] for the moduli spaces of $G$-constellations and the corresponding tautological free sheaves discussed in Section 2.

It is a useful heuristic to think of bundles with decaying connections as bundles on a compactification whose restrictions to the "divisor at infinity" satisfy certain "vanishing conditions". With this in mind, we compactify $M_{\theta}$ at infinity by gluing $M_{\theta}$ and $\left(\mathbb{P}^{3} \backslash\{[0: 0: 0: 1]\}\right) / G$ along $M_{\theta} \backslash \pi_{\theta}^{-1}(0)=\left(\mathbb{C}^{3} \backslash\{0\}\right) / G$. The resulting space $\bar{M}_{\theta}$ is not a complex manifold, but rather a complex orbifold. One can think of $\bar{M}_{\theta}$ as obtained from $M_{\theta}$ by adjoining the divisor $D=\mathbb{P}^{2} / G$ at infinity. $D$ is a smooth orbifold divisor, i.e., it lifts to a smooth divisor in covers of the uniformising charts. The holomorphic bundle $\mathcal{R}$ extends over $D$ to a holomorphic bundle $\overline{\mathcal{R}}$ on $\bar{M}_{\theta}$. The following result reduces the proof of Lemma 5.1 to a problem in complex geometry.

Proposition 5.2. $\mathcal{H}_{A}^{1}$ injects into $H^{1}\left(\bar{M}_{\theta}, \mathcal{E} \operatorname{nd}(\overline{\mathcal{R}})(-D)\right)$.

Recall that for a holomorphic vector bundle $\mathcal{E}, \mathcal{E}(-D)$ is the sheaf of holomorphic sections of $\mathcal{E}$ vanishing to first order along $D$. The proof of Proposition 5.2 requires two preparatory results.

Proposition 5.3. Let $Z$ be a complex orbifold, $D$ be a smooth divisor in $Z$ and $\mathcal{E}$ be a holomorphic bundle on $Z$. Denote by $i: D \hookrightarrow Z$ the inclusion of $D$ into $Z$. Then the complex of sheaves $\left(\mathcal{A}^{\bullet}, \bar{\partial}\right)$ defined by

$$
\mathcal{A}^{k}(U):=\left\{\alpha \in \Omega^{0, k}(U, \mathcal{E}): i^{*} \alpha=0\right\}
$$

for $U \subset Z$ open is an acyclic resolution of $\mathcal{E}(-D)$.

Proof. Since $i^{*}$ and $\bar{\partial}$ commute, $\mathcal{A}^{\bullet}$ forms a complex. Moreover, it is clear that $\mathcal{E}(-D)$ is the kernel of $\mathcal{A}^{0} \stackrel{\bar{\partial}}{\rightarrow} \mathcal{A}^{1}$.

The proof that $\left(\mathcal{A}^{\bullet}, \bar{\partial}\right)$ is a resolution uses two ingredients: the Grothendieck-Dolbeault lemma and the fact that if $U$ is a sufficiently small open set, then holomorphic sections on $D \cap U$ extend to $U$. First we show that these assertions also hold for orbifolds. Let $U$ be a small open set which is covered by a uniformising chart $\tilde{U} / \Gamma$ where $\Gamma$ is a finite group. Lifting everything up to $\tilde{U}, \mathcal{E}$ corresponds to a $\Gamma$-equivariant holomorphic bundle $\tilde{\mathcal{E}}$ and $D$ to a $\Gamma$-equivariant smooth divisor $\tilde{D}$. If $\alpha \in \Omega^{0, k}(U, \mathcal{E})$ satisfies $\bar{\partial} \alpha=0$, then so does its lift $\tilde{\alpha} \in \Omega^{0, k}(\tilde{U}, \tilde{\mathcal{E}})^{\Gamma}$. If $U$ (and thus $\tilde{U}$ ) is sufficiently small, then the usual Grothendieck-Dolbeault lemma yields $\tilde{\beta} \in \Omega^{0, k-1}(\tilde{U}, \tilde{\mathcal{E}})$ satisfying $\bar{\partial} \tilde{\beta}=\tilde{\alpha}$. There is no loss in assuming that $\tilde{\beta}$ is $\Gamma$-invariant and thus pushes down to the desired primitive $\beta \in \Omega^{0, k-1}(U, \mathcal{E})$ of $\alpha$. We thus obtain the GrothendieckDolbeault lemma for orbifolds. Now, if $s$ is a holomorphic section of $\mathcal{E}$ over $D \cap U$, we lift it to the uniformising chart $\tilde{U}$, where, provided $U$ is sufficiently small, we find a $\Gamma$-equivariant extension. We then push this extension down to $U$. This proves the second assertion.

Let now $U$ be a small open set of $Z$ and let $\alpha \in \Omega^{0, k}(U, \mathcal{E})$ with $\bar{\partial} \alpha=0$. By the GrothendieckDolbeault lemma after possibly shrinking $U$, we can find $\beta \in \Omega^{0, k-1}(U, \mathcal{E})$ satisfying $\bar{\partial} \beta=\alpha$. If $k \geq 2$, we apply the Grothendieck-Dolbeault lemma once more to obtain $\gamma \in \Omega^{0, k-2}(U \cap D, \mathcal{E})$ such that $\bar{\partial} \gamma=i^{*} \beta$. We extend $\gamma$ smoothly to all of $U$. Then $\beta-\bar{\partial} \gamma \in \mathcal{A}^{k-1}(U)$ yields the desired primitive of $\alpha$ on $U$. If $k=1$, we know that $\beta$ restricts to a holomorphic section $\left.\beta\right|_{D}$ of $\left.\mathcal{E}\right|_{U \cap D}$, which can be extended to a holomorphic section $\delta$ on $U$. Hence, $\beta-\delta \in \mathcal{A}^{0}(U)$ is the desired primitive of $\alpha$.

Finally, $\left(\mathcal{A}^{\bullet}, d\right)$ is an acyclic resolution of $\mathcal{E}(-D)$, since the sheaves $\mathcal{A}^{\bullet}$ are $C^{\infty}$-modules and therefore soft.

Remark 5.4. In the definition of $\mathcal{A}^{k}$ it is not strictly necessary to require that $\alpha$ be smooth. In fact, a simple application of elliptic regularity shows that it suffices that elements of $\mathcal{A}^{k}$ be in the Hölder space $C^{n-k, \gamma}$, where $n$ denotes the complex dimension of $Z$. 
Proposition 5.5. If $a \in \mathcal{H}_{A}^{1}$, then

$$
\nabla_{A}^{k} a=O\left(r^{-5-k}\right) \quad \text { for all } k \geq 0 .
$$

Proof. First observe that using simple scaling considerations and standard elliptic theory, (5.1) for $k>0$ follows from the case $k=0$.

It is rather straightforward to obtain $a=O\left(r^{-4}\right)$ using the maximum principle. To obtain the stronger decay estimate it is customary to use a refined Kato inequality, see, e.g., Bando, Kasue and Nakajima [3]. Recall that the classical Kato inequality is a consequence of the CauchySchwarz inequality $\left|\left\langle\nabla_{A} a, a\right\rangle\right| \leq\left|\nabla_{A} a\right||a|$. In our case, since $a$ is not arbitrary but satisfies $\bar{\partial} a=\bar{\partial}_{A}^{*} a=0$, the above inequality can be improved upon: There exists a constant $\gamma<1$ such that $|\mathrm{d}| a|| \leq \gamma\left|\nabla_{A} a\right|$ on the set $U:=\left\{x \in M_{\theta}: a(x) \neq 0\right\}$. A detailed analysis shows that since we are working on a 6 -dimensional real manifold, we can choose $\gamma$ to be $\sqrt{5 / 6}$, see, e.g., [9].

We set $\gamma:=\sqrt{5 / 6}$ and let $\sigma:=2-1 / \gamma^{2}=4 / 5$. Using the refined Kato inequality for $a$, we obtain

$$
\begin{aligned}
(2 / \sigma) \Delta|a|^{\sigma} & =|a|^{\sigma-2}\left(\Delta|a|^{2}-2(\sigma-2)|\mathrm{d}| a||^{2}\right) \\
& \leq|a|^{\sigma-2}\left(\Delta|a|^{2}+2\left|\nabla_{A} a\right|^{2}\right)=|a|^{\sigma-2}\left\langle a, \nabla_{A}^{*} \nabla_{A} a\right\rangle
\end{aligned}
$$

on $U$. The Weitzenböck formula for $\nabla_{A}^{*} \nabla_{A} a$ gives

$$
(2 / \sigma) \Delta|a|^{\sigma} \leq|a|^{\sigma-2}\left(\left\langle\Delta_{\bar{\partial}_{A}} a, a\right\rangle+\langle\{\text { Riem, } a\}, a\rangle+\left\langle\left\{F_{A}, a\right\}, a\right\rangle\right),
$$

with Riem the Riemannian curvature and $F_{A}$ the curvature of the connection $A$. Because $\Delta_{\bar{\partial}_{A}} a=$ 0 and since by hypothesis of Lemma 5.1 the metric on $M_{\theta}$ is ALE and the connection $A$ is asymptotically flat, there exist constants $c, \beta>0$ so that on $U$ we have

$$
(2 / \sigma) \Delta|a|^{\sigma} \leq c(1+r)^{-2-\beta}|a|^{\sigma} .
$$

Set $f:=|a|^{\sigma}$. We show that $f=O\left(r^{-4}\right)$, which is equivalent to the desired decay estimate for $a$. Note that on $U$ the above estimate gives

$$
\Delta f \leq \frac{c f}{1+r^{2+\beta}}
$$

Since $f$ decays at infinity and is thus bounded, using the method of proof of [19, Theorem 8.3.6(a)], there exists $g \in C^{2, \alpha}$ with $g=O\left(r^{-\beta}\right)$ such that

$$
\Delta g= \begin{cases}(\Delta f)^{+} & \text {on } U, \\ 0 & \text { on } M_{\theta} \backslash U .\end{cases}
$$

Here $h^{+}$denotes the positive part of the function $h$, i.e., $h^{+}:=\max \{h, 0\}$. Since $g$ is superharmonic and decays to zero at infinity, the maximum principle implies that $g$ is non-negative on the boundary of $U$; hence, by the maximum principle $f \leq g=O\left(r^{-\beta}\right)$. By (5.2) we then have $(\Delta f)^{+}=O\left(r^{-2-2 \beta}\right)$, which then yields that $f=O\left(r^{-2 \beta}\right)$. Iterating this argument $k$ times we obtain $f=O\left(r^{-k \beta}\right)$ for all $k<(n-2) / \beta$ with $n=6$ the real dimension of $M_{\theta}$. For the biggest $k$ with this property, we have $2+(k+1) \beta>n$. Then, by [19, Theorem 8.3.6(b)], we can chose $g$ above such that $g=O\left(r^{-4}\right)$. Therefore, $f=O\left(r^{-4}\right)$ as desired.

With these two preliminary results, we can now prove Proposition 5.2. 
Proof of Proposition 5.2. Given $a \in \mathcal{H}_{A}^{1}$, we extend it to a 1 -form on $\bar{M}_{\theta}$ vanishing along $D$. From Proposition 5.5 it follows that $a$ vanishes to third order along $D$. Hence, $a$ is in $C^{2, \alpha}\left(\bar{M}_{\theta}\right)$ and we can regard it as an element of $\mathcal{A}^{1}\left(\bar{M}_{\theta}\right)$. Since $\bar{\partial} a=0$, by Proposition 5.3 it gives an element $[a] \in H^{1}\left(\bar{M}_{\theta}, \mathcal{E} \operatorname{nd}(\overline{\mathcal{R}})(-D)\right)$. This defines a linear map $i: \mathcal{H}_{A}^{1} \rightarrow H^{1}\left(\bar{M}_{\theta}, \mathcal{E} \operatorname{nd}(\overline{\mathcal{R}})(-D)\right)$.

We will now show that $i$ is injective. Suppose that $i(a)=0$, i.e., there exists $b \in \mathcal{A}^{0}\left(\bar{M}_{\theta}\right)$ so that $a=\bar{\partial} b$. Since $b$ vanishes along $D$, its restriction to $M_{\theta}$ decays like $r^{-1}$. Using this together with $a=O\left(r^{-5}\right)$, we can integrate by parts to obtain

$$
\|a\|_{L^{2}}^{2}=\int_{M_{\theta}}\langle a, \bar{\partial} b\rangle \mathrm{dvol}_{g}=\int_{M_{\theta}}\left\langle\bar{\partial}_{A}^{*} a, b\right\rangle \operatorname{dvol}_{g}=0 .
$$

It follows that $a$ vanishes, and thus $i$ is injective.

To prove Lemma 5.1 it now suffices to establish the following result:

Proposition 5.6. $H^{1}\left(\bar{M}_{\theta}, \mathcal{E} \operatorname{nd}(\overline{\mathcal{R}})(-D)\right)=0$.

To prove this statement, we convert it into a problem in algebraic geometry. In the same way we compactified $M_{\theta}$, we can complete the scheme $\mathscr{M}_{\theta}$ at infinity by attaching $D=\mathbb{P}^{2} / G$. This yields an algebraic stack $\overline{\mathfrak{M}}_{\theta}$. Moreover, $\mathscr{R}$ extends to a locally free sheaf $\overline{\mathscr{R}}$ on $\overline{\mathfrak{M}}_{\theta}$. By GAGA [27, Théorème 5.10], Proposition 5.6 is equivalent to

$$
H^{1}\left(\overline{\mathfrak{M}}_{\theta}, \mathscr{E} \operatorname{nd}(\mathscr{R})(-D)\right)=0 .
$$

To establish this we need the following consequence of Theorem 2.6.

Proposition 5.7. For generic $\theta \in \Theta_{\mathbb{Q}}$,

$$
H^{k}\left(\mathscr{M}_{\theta}, \mathscr{R}_{\rho}^{*} \otimes \mathscr{R}_{\sigma}\right)=H^{k}\left(\mathbb{C}^{3}, \mathscr{O} \otimes R_{\rho}^{*} \otimes R_{\sigma}\right)^{G},
$$

for all $\rho, \sigma \in \operatorname{Irr}(G)$. In particular, for $k>0$,

$$
H^{k}\left(\mathscr{M}_{\theta}, \mathscr{R}_{\rho}^{*} \otimes \mathscr{R}_{\sigma}\right)=0 .
$$

If $G$ acts freely on $\mathbb{C}^{3} \backslash\{0\}$, we have a commutative diagram

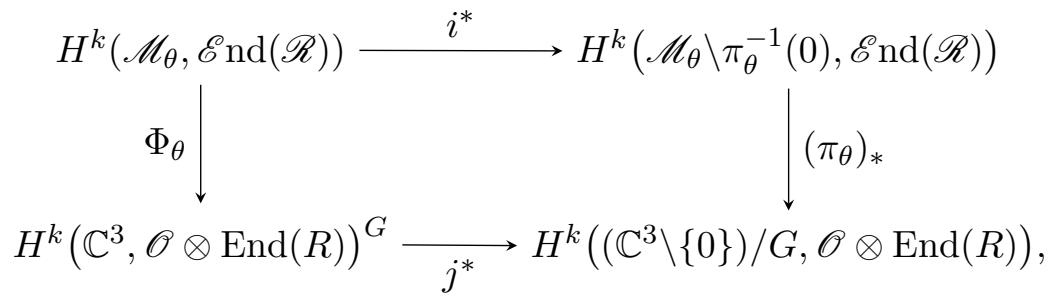

where $i: \mathscr{M}_{\theta} \backslash \pi_{\theta}^{-1}(0) \rightarrow \mathscr{M}_{\theta}$ and $j: \mathbb{C}^{3} \backslash\{0\} \rightarrow \mathbb{C}^{3}$ are the inclusion maps.

Proof. The first part is due to Craw and Ishii [10, Lemma 5.4]. Let us briefly recall their proof. We have

$$
H^{k}\left(\mathscr{M}_{\theta}, \mathscr{R}_{\rho}^{*} \otimes \mathscr{R}_{\sigma}\right)=\operatorname{Ext}^{k}\left(\mathscr{O}, \mathscr{R}_{\rho}^{*} \otimes \mathscr{R}_{\sigma}\right)=\operatorname{Ext}^{k}\left(\mathscr{R}_{\rho}, \mathscr{R}_{\sigma}\right)=H^{k}\left(\operatorname{Hom}_{D\left(\mathscr{M}_{\theta}\right)}\left(\mathscr{R}_{\rho}, \mathscr{R}_{\sigma}\right)\right)
$$

and

$$
\begin{aligned}
H^{k}\left(\mathbb{C}^{3}, \mathscr{O} \otimes R_{\rho}^{*} \otimes R_{\sigma}\right)^{G} & =G-\operatorname{Ext}^{k}\left(\mathscr{O} \otimes R_{\rho}, \mathscr{O} \otimes R_{\sigma}\right) \\
& =H^{k}\left(\operatorname{Hom}_{D^{G}\left(\mathbb{C}^{3}\right)}\left(\mathscr{O} \otimes R_{\rho}, \mathscr{O} \otimes R_{\sigma}\right)\right) .
\end{aligned}
$$


Moreover, the inverse of the Fourier-Mukai transform $\Phi_{\theta}$ is given by

$$
\Phi_{\theta}^{-1}=\left(p_{*}\left(q^{*}(-) \otimes \mathscr{U}_{\theta}^{D}[3]\right)\right)^{G}=\left(-\otimes \bigoplus_{\rho} \mathscr{R}_{\rho}^{*} \otimes R_{\rho}\right)^{G}
$$

see $\left[10\right.$, p. 267]. Here $(-)^{D}$ denotes the derived dual. In particular,

$$
\Phi_{\theta}^{-1}\left(\mathscr{O} \otimes R_{\rho}\right)=\mathscr{R}_{\rho} .
$$

Therefore, according to Theorem 2.6,

$$
\begin{aligned}
H^{k}\left(\mathscr{M}_{\theta}, \mathscr{R}_{\rho}^{*} \otimes \mathscr{R}_{\sigma}\right) & =H^{k}\left(\operatorname{Hom}_{D\left(\mathscr{M}_{\theta}\right)}\left(\mathscr{R}_{\rho}, \mathscr{R}_{\sigma}\right)\right) \\
& \cong H^{k}\left(\operatorname{Hom}_{D^{G}\left(\mathbb{C}^{3}\right)}\left(\mathscr{O} \otimes R_{\rho}, \mathscr{O} \otimes R_{\sigma}\right)\right)=H^{k}\left(\mathbb{C}^{3}, \mathscr{O} \otimes R_{\rho}^{*} \otimes R_{\sigma}\right)^{G} .
\end{aligned}
$$

To prove the second part, we show the commutativity of the diagram

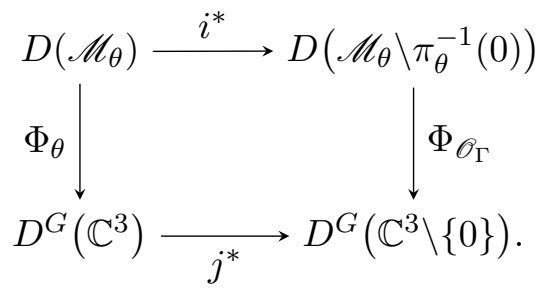

Here $\Phi_{\mathscr{O}_{\Gamma}}$ is the Fourier-Mukai transform with kernel $\mathscr{O}_{\Gamma}$, the structure sheaf of the graph of $\pi_{\theta}: \mathscr{M}_{\theta} \backslash \pi_{\theta}^{-1}(0) \rightarrow \mathbb{C}^{3} \backslash\{0\}$. Note that under the identification $D^{G}\left(\mathbb{C}^{3} \backslash\{0\}\right)=D\left(\left(\mathbb{C}^{3} \backslash\{0\}\right) / G\right)$, $\Phi_{\mathscr{O}_{\Gamma}}$ becomes $\left(\pi_{\theta}\right)_{*}$.

Denote by $r$ and $s$ the projections from $\mathscr{M}_{\theta} \backslash \pi_{\theta}^{-1}(0) \times \mathbb{C}^{3} \backslash\{0\}$ to $\mathscr{M}_{\theta} \backslash \pi_{\theta}^{-1}(0)$ and $\mathbb{C}^{3} \backslash\{0\}$, respectively. Let $t: \mathscr{M}_{\theta} \times \mathbb{C}^{3} \backslash\{0\} \rightarrow \mathbb{C}^{3} \backslash\{0\}$ denote the projection onto the second factor. The following diagram summarises the situation:

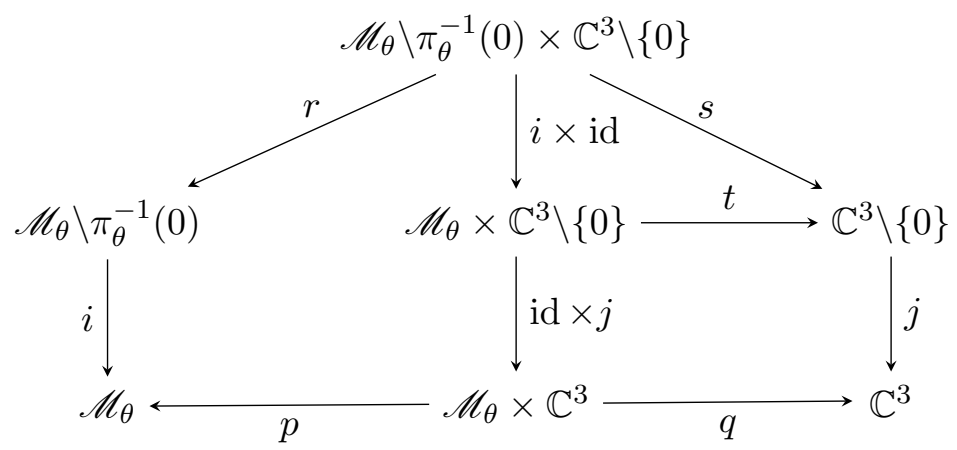

It follows, essentially from the definition of $\pi_{\theta}$, that

$$
\left(\operatorname{id} \mathscr{M}_{\theta} \times j\right)^{*} \mathscr{U}_{\theta}=\left(i \times \operatorname{id}_{\mathbb{C}^{3} \backslash\{0\}}\right)_{*} \mathscr{O}_{\Gamma} .
$$

Using (5.4) as well as the push-pull formula $\left(\mathscr{F} \otimes f_{*} \mathscr{G}\right)=f_{*}\left(f^{*} \mathscr{F} \otimes \mathscr{G}\right)$ we obtain

$$
\begin{aligned}
j^{*} \circ \Phi_{\theta}(-) & =j^{*} \circ q_{*}\left(p^{*}\left(-\otimes \rho_{0}\right) \otimes \mathscr{U}_{\theta}\right)=t_{*}\left(\left(\operatorname{id}_{\mathscr{M}_{\theta}} \times j\right)^{*} p^{*}\left(-\otimes \rho_{0}\right) \otimes\left(\operatorname{id}_{\mathscr{M}_{\theta}} \times j\right)^{*} \mathscr{U}_{\theta}\right) \\
& =t_{*}\left(\left(\operatorname{id}_{\mathscr{M}_{\theta}} \times j\right)^{*} p^{*}\left(-\otimes \rho_{0}\right) \otimes\left(i \times \operatorname{id}_{\mathbb{C}^{3} \backslash\{0\}}\right)_{*} \mathscr{O}_{\Gamma}\right) \\
& =t_{*}\left(i \times \operatorname{id}_{\mathbb{C}^{3} \backslash\{0\}}\right)_{*}\left((i \times j)^{*} p^{*}\left(-\otimes \rho_{0}\right) \otimes \mathscr{O}_{\Gamma}\right) \\
& =s_{*}\left(r^{*} i^{*}\left(-\otimes \rho_{0}\right) \otimes \mathscr{O}_{\Gamma}\right)=\Phi_{\mathscr{O}_{\Gamma}} \circ i^{*}(-) .
\end{aligned}
$$

This concludes the proof. 
Before we embark on the proof of (5.3), it is useful to recall some basic properties of local cohomology, see, e.g., [15, Chapter III, Exercise 2.3]. Let $D$ be a closed subset of $X$ and let $\mathcal{E}$ be a sheaf on $X$. Denote by $\Gamma_{D}(X, \mathscr{E})$ the subspace of $\Gamma(X, \mathscr{E})$ consisting of sections whose support is contained in $D$. The functor $\Gamma_{D}(X,-)$ is left-exact and its right derived functor $H_{D}^{\bullet}(X, \mathscr{E}):=R^{\bullet} \Gamma_{D}(X, \mathscr{E})$ is called the local cohomology of $\mathcal{E}$ with respect to $D$. Local cohomology is related to the usual cohomology of $\mathcal{E}$ by the following long exact sequence

$$
\cdots \rightarrow H_{D}^{i}(X, \mathscr{E}) \rightarrow H^{i}(X, \mathscr{E}) \rightarrow H^{i}\left(X \backslash D,\left.\mathscr{E}\right|_{X \backslash D}\right) \stackrel{\delta}{\rightarrow} H_{D}^{i+1}(X, \mathscr{E}) \rightarrow \cdots
$$

Moreover, it satisfies excision, that is, if $U$ is an open subset in $X$ containing $D$, then there is a natural isomorphism

$$
H_{D}^{\bullet}(X, \mathcal{E}) \cong H_{D}^{\bullet}\left(U,\left.\mathcal{E}\right|_{U}\right) .
$$

Proof of Proposition 5.6. We have already reduced the proof of this to the proof of the vanishing statement (5.3). Since by Proposition 5.7 we have $H^{1}\left(\mathscr{M}_{\theta}, \mathscr{E} \mathrm{nd}(\mathscr{R})\right)=0$, the long exact sequence associated to local cohomology yields

$$
H^{0}\left(\mathscr{M}_{\theta}, \mathscr{E} \operatorname{nd}(\mathscr{R})\right) \stackrel{\delta}{\rightarrow} H_{D}^{1}\left(\overline{\mathfrak{M}}_{\theta}, \mathscr{E} \operatorname{nd}(\overline{\mathscr{R}})(-D)\right) \rightarrow H^{1}\left(\overline{\mathfrak{M}}_{\theta}, \mathscr{E} \operatorname{nd}(\overline{\mathscr{R}})(-D)\right) \rightarrow 0 .
$$

We show that the first map in this sequence is an isomorphism. This gives the desired vanishing: $H^{1}\left(\overline{\mathfrak{M}}_{\theta}, \mathscr{E} \operatorname{nd}(\overline{\mathscr{R}})(-D)\right)=0$.

Let $H$ denote the hyperplane section in $\mathbb{P}^{3}$. By excision, we have

$$
\begin{aligned}
H_{D}^{1}\left(\overline{\mathfrak{M}}_{\theta}, \mathscr{E} \operatorname{nd}(\overline{\mathscr{R}})(-D)\right) & \cong H_{D}^{1}\left(\overline{\mathfrak{M}}_{\theta} \backslash \pi^{-1}(0), \mathscr{E} \operatorname{nd}(\overline{\mathscr{R}})(-D)\right) \\
& =H_{H}^{1}\left(\left[\mathbb{P}^{3} \backslash\{[0: 0: 0: 1]\} / G\right], \mathscr{O}(-1) \otimes \operatorname{End}(R)\right) \\
& \cong H_{H}^{1}\left(\left[\mathbb{P}^{3} / G\right], \mathscr{O}(-1) \otimes \operatorname{End}(R)\right) .
\end{aligned}
$$

Here and in the following we omit to make the appropriate restriction of sheaves explicit, because confusion is unlikely to arise. Using the above, we have the commutative diagram

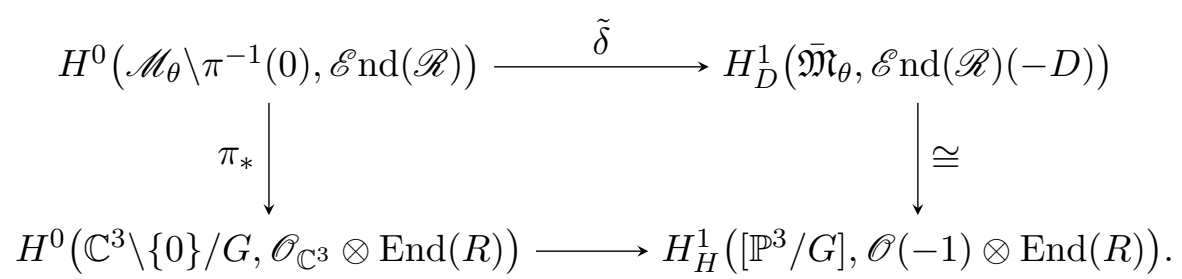

We compose on the left with the commutative diagram in Proposition 5.7. Since $\delta=\tilde{\delta} \circ i^{*}$, we obtain the commutative diagram

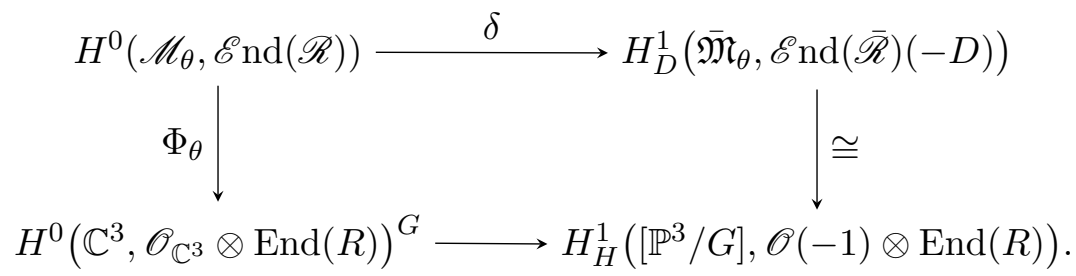

All the vertical arrows are isomorphisms. Moreover, by using the long exact sequence (5.5) and

$$
H^{i}\left(\left[\mathbb{P}^{3} / G\right], \mathscr{O}(-1) \otimes \operatorname{End}(R)\right)=H^{i}\left(\mathbb{P}^{3}, \mathscr{O}(-1) \otimes \operatorname{End}(R)\right)^{G}=0
$$

for $i=0$ and 1 , it follows that the bottom map in the above diagram is an isomorphism. Therefore, the map $\delta$ must also be an isomorphism. 


\section{Dirac operators on $M_{\theta}$}

Let $(X, g)$ be an ALE spin manifold asymptotic to $\mathbb{C}^{n} / G$ and let $E$ be a complex vector bundle over $X$ together with an asymptotically flat connection $A$. Denote by $S^{ \pm}$be the spinor bundles on $X$ and by $D_{E}^{ \pm}$the corresponding twisted Dirac operators. We denote by $W_{\delta}^{k, 2}\left(X, S^{ \pm} \otimes E\right)$ the completions of the spaces of compactly supported sections with respect the weighted Sobolev norm defined by (4.1) using the covariant derivative $\nabla_{A}$. Let $D_{E, \delta}^{ \pm}: W_{\delta}^{k+1,2}\left(X, S^{ \pm} \otimes E\right) \rightarrow$ $W_{\delta-1}^{k, 2}\left(Z, S^{\mp} \otimes E\right)$ denote the corresponding completion of the Dirac operator $D_{E}^{ \pm}$.

Theorem 6.1. For $\delta \in(-2 n-1,0)$ the Dirac operator $D_{E, \delta}^{ \pm}$is Fredholm and its index is given by

$$
\operatorname{index} D_{E, \delta}^{+}=\int_{X} \operatorname{ch}(E) \hat{A}(X)-\frac{\eta_{E}}{2} .
$$

Here $\operatorname{ch}(E)$ denotes the Chern character of $E$ as a differential form, $\hat{A}(X)$ denotes the Hirzebruch A-polynomial applied to the Pontryagin forms $p_{i}(X)$ of the ALE metric on $X, \eta_{E}(s):=$ $\sum_{\lambda \neq 0} \operatorname{sign}(\lambda)|\lambda|^{-s}$ is the $\eta$-function of the spectrum of the Dirac operator restricted to the boundary at infinity $S^{2 n-1} / G$ of the ALE manifold $X$, and $\eta_{E}:=\eta_{E}(0)$ is the $\eta$-invariant.

Proof. The fact that $D_{E, \delta}^{ \pm}$is Fredholm is proved as in Proposition 4.10 by noting that the set of indicial roots does not intersect $(-2 n-1,0)$. This can be seen, for example, by realizing that the indicial roots correspond to the eigenvalues of the Dirac operator on $S^{2 n-1} / G$ shifted by $-\frac{2 n-1}{2}$. The index formula follows from Atiyah-Patodi-Singer index theorem [1].

If $(X, g)$ is a Kähler manifold, then there is a one-to-one correspondence between spin structures on $X$ and holomorphic square roots of the canonical line bundle $K_{X}$, see [16, Theorem 2.2]. We are interested in the case when $X=M_{\theta}$ for $\theta \in \Theta_{\mathbb{Q}}$ a generic stability parameter. Since $M_{\theta}$ is a crepant resolution of $\mathbb{C}^{3} / G$, its canonical line bundle is holomorphically trivial. In particular, for any Kähler metric on $M_{\theta}$ there is a canonical spin structure corresponding to taking the square root of the canonical bundle to be the trivial holomorphic line bundle on $M_{\theta}$. The corresponding spinor bundles are

$$
S^{+}=\Lambda^{0, \text { even }} T_{\mathbb{C}}^{*} M_{\theta} \quad \text { and } \quad S^{-}=\Lambda^{0, \text { odd }} T_{\mathbb{C}}^{*} M_{\theta} .
$$

We fix this spin structure on $M_{\theta}$ for the rest of the section. Suppose that $A$ is a $\mathrm{U}(n)$-connection on a holomorphic bundle $\mathcal{E}$ compatible with the given holomorphic structure. If the metric on $M_{\theta}$ induces the product connection on our chosen square root of $K_{M_{\theta}}$, i.e., the metric is Ricci-flat, then the corresponding twisted Dirac operator is given by

$$
D_{\mathcal{E}}^{ \pm}=\sqrt{2}\left(\bar{\partial}_{A}+\bar{\partial}_{A}^{*}\right) .
$$

We now show that for special choices of $\mathcal{E}$ involving the tautological bundles on $M_{\theta}$, the index of the corresponding weighted Dirac operator is zero. Recall that by Theorem 1.1, there exists an ALE Calabi-Yau metric $g_{\theta, \mathrm{RF}}$ on $M_{\theta}$ in the Kähler class of $g_{\theta}$ and a HYM connection on each of the tautological holomorphic line bundles $\mathcal{R}_{\rho}$ with $\rho \in \operatorname{Irr}(G)$. Moreover these induce a HYM connection $A$ on $\mathcal{R}=\bigoplus_{\rho \in \operatorname{Irr}(G)} \mathcal{R}_{\rho}$ which is infinitesimally rigid.

Proposition 6.2. Let $\theta \in \Theta_{\mathbb{Q}}$ be generic.

(1) If $g$ is an ALE Kähler metric on $M_{\theta}$ in the same Kähler class as $g_{\theta}$, then

$$
\text { index } D_{\mathcal{R}_{\rho} \otimes \mathcal{R}_{\sigma}^{*}, \delta}^{+}=0,
$$

for all $\rho, \sigma \in \operatorname{Irr}(G)$ and for all $\delta \in(-5,0)$. 
(2) If $g=g_{\theta, \mathrm{RF}}$ and $\mathcal{R}$ is equipped with the HYM connection A given by Theorem 1.1(3), then the twisted Dirac operator $D_{\mathcal{E} \mathrm{nd}(\mathcal{R}), \delta}^{+}$is an isomorphism for all $\delta \in(-5,0)$.

Proof. The index of $D_{\mathcal{R}_{\rho} \otimes \mathcal{R}_{\sigma}^{*}, \delta}$ is unchanged under deforming the ALE Kähler metric and the asymptotically flat connection on $\mathcal{R}_{\rho} \otimes \mathcal{R}_{\sigma}^{*}$. Hence there is no loss in assuming that $g=g_{\theta, \mathrm{RF}}$ and that $\mathcal{R}_{\rho} \otimes \mathcal{R}_{\sigma}^{*}$ has been equipped with its HYM metric. Since the twisted Dirac operators preserve the holomorphic splitting $\mathcal{E} \operatorname{nd}(\mathcal{R})=\bigoplus_{\rho, \sigma \in \operatorname{Irr}(G)} \mathcal{R}_{\rho}^{*} \otimes \mathcal{R}_{\sigma}$, we are thus left with proving the second statement.

First we prove that index $D_{\mathcal{E} \text { nd }(\mathcal{R}), \delta}^{+}=0$. Let $\Omega$ be a nowhere vanishing holomorphic volume form on $M_{\theta}$ and let $*: \Lambda^{p, q} T^{*} M_{\theta} \otimes \mathcal{E} \rightarrow \Lambda^{3-p, 3-q} T^{*} M_{\theta} \otimes \mathcal{E}^{*}$ denote the Hodge-*-operator for some holomorphic bundle $\mathcal{E}$. Then we have an isomorphism of vector bundles $S^{+} \otimes \mathcal{E} \cong S^{-} \otimes \mathcal{E}^{*}$, given by

$$
\begin{aligned}
S^{+} \otimes \mathcal{E} & =\Lambda^{0, \text { even }} T^{*} M_{\theta} \otimes \mathcal{E} \stackrel{\Omega \wedge-}{\longrightarrow} \Lambda^{3, \text { even }} T^{*} M_{\theta} \otimes \mathcal{E} \\
& \stackrel{*}{\longrightarrow} \Lambda^{0, \text { odd }} T^{*} M_{\theta} \otimes \mathcal{E}^{*}=S^{-} \otimes \mathcal{E}^{*} .
\end{aligned}
$$

Similarly, $S^{-} \otimes \mathcal{E} \cong S^{+} \otimes \mathcal{E}^{*}$. These isomorphisms identify $D_{\mathcal{E}}^{+}$with $D_{\mathcal{E}^{*}}^{-}$. Consequently

$$
\operatorname{index} D_{\mathcal{E}, \delta}^{+}=\operatorname{index} D_{\mathcal{E}^{*}, \delta}^{-} \text {. }
$$

Moreover, the $L^{2}$-adjoint of $D_{\mathcal{E}^{*}, \delta}^{-}$is $D_{\mathcal{E}^{*},-5-\delta}^{+}$and thus

$$
\operatorname{index} D_{\mathcal{E}^{*}, \delta}^{-}=-\operatorname{index} D_{\mathcal{E}^{*},-5-\delta}^{+} \text {. }
$$

For $\mathcal{E}=\mathcal{E}$ nd $(\mathcal{R})$ and $\delta=-\frac{5}{2}$, the two identities above give that index $D_{\mathcal{E} \text { nd }(\mathcal{R}),-\frac{5}{2}}^{+}=0$. Since the index is constant for $\delta \in(-5,0)$, we must have index $D_{\mathcal{E} \text { nd }(\mathcal{R}), \delta}^{+}=0$ for all $\delta \in(-5,0)$.

To complete the proof, we show that coker $D_{\mathcal{E} \text { nd }(\mathcal{R}), \delta}^{+}=0$ for $\delta \in(-5,0)$ or, equivalently, that ker $D_{\mathcal{E} \text { nd }(\mathcal{R}),-5-\delta}^{-}=0$. By abuse of notation we also denote by $A$ the connection induced by $A$ on $\mathcal{E} \operatorname{nd}(\mathcal{R})$. Each $\left(\phi_{1}, \phi_{3}\right) \in \operatorname{ker} D_{\mathcal{E} \text { nd }(\mathcal{R})}^{-}$satisfies

$$
\bar{\partial}_{A}^{*} \phi_{1}=0 \quad \text { and } \quad \bar{\partial}_{A} \phi_{1}+\bar{\partial}_{A}^{*} \phi_{3}=0
$$

The second identity gives

$$
\bar{\partial}_{A} \bar{\partial}_{A}^{*} \phi_{3}=0
$$

Arguing as in Proposition 5.5 one shows that $\phi_{3}=O\left(r^{-4}\right)$ and $\bar{\partial}_{A}^{*} \phi_{3}=O\left(r^{-5}\right)$. Hence, taking the $L^{2}$-inner-product with $\phi_{3}$ in (6.4) and integrating by parts, we conclude that $\bar{\partial}_{A}^{*} \phi_{3}=0$. On the other hand, we have the splitting $\nabla_{A}=\partial_{A}+\bar{\partial}_{A}$ and the Kähler identity gives $\partial_{A}=i\left[\Lambda, \bar{\partial}_{A}^{*}\right]$. Using this, the above yields $\partial_{A} \phi_{3}=0$. If we write $\phi_{3}=\bar{\Omega} \otimes s$ with $s$ a smooth section of $\mathcal{E}$ nd $(\mathcal{R})$, then $\partial_{A}(\bar{\Omega} \otimes s)=\bar{\Omega} \wedge \partial_{A} s=0$ and thus $\partial_{A} s=0$. Since the connection $A$ on $\mathcal{R}$ is HYM and $g_{\theta, \mathrm{RF}}$ is Ricci-flat, the Weitzenböck formula gives $2 \Delta_{\partial_{A}}=\nabla_{A}^{*} \nabla_{A}$. It follows that $s$ is parallel and hence must vanish, as it is zero at infinity. This implies that $\phi_{3}=0$. From (6.3) we deduce that $\phi_{1}$ satisfies $\bar{\partial}_{A} \phi_{1}=0$ and $\bar{\partial}_{A}^{*} \phi_{1}=0$. Then, by Lemma 5.1, $\phi_{1}$ must vanish identically.

\section{The proof of Theorem 1.4}

We now prove Theorem 1.4. We start by discussing the sense in which formula (1.2) is valid. Since $M_{\theta}$ is non-compact, this formula cannot be interpreted as a difference of triple products in $H^{*}\left(M_{\theta}, \mathbb{R}\right)$ as $H^{6}\left(M_{\theta}, \mathbb{R}\right)=0$. 
However, each of the tautological line bundles $\mathcal{R}_{\rho}$ have natural asymptotically flat connections $A_{\theta, \rho}$ compatible with their holomorphic structure. Via Chern-Weil theory, $c_{1}\left(\mathcal{R}_{\rho}\right)$ is represented in $H^{2}\left(M_{\theta}, \mathbb{R}\right)$ by the de Rham cohomology class of $\frac{i}{2 \pi} F_{A_{\theta, \rho}}$. These are the differential forms used in the Atiyah-Patodi-Singer formula (6.1) to represent the Chern character of the bundle $\mathcal{R}_{\rho}$. Using this formula we will show below how to obtain (1.2) (in fact, the equivalent formulation (1.3)) at the level of differential forms representing the first Chern classes.

Moreover, since we are on an ALE manifold, the long exact sequence in cohomology

$$
\cdots \rightarrow H^{1}\left(S^{5} / G, \mathbb{R}\right) \rightarrow H_{c}^{2}\left(M_{\theta}, \mathbb{R}\right) \stackrel{j}{\rightarrow} H^{2}\left(M_{\theta}, \mathbb{R}\right) \rightarrow H^{2}\left(S^{5} / G, \mathbb{R}\right) \rightarrow \cdots
$$

gives that the homomorphism $j$, which takes the class of a compactly supported form in $H_{c}^{2}\left(M_{\theta}, \mathbb{R}\right)$ to its de Rham representative in $H^{2}\left(M_{\theta}, \mathbb{R}\right)$, is an isomorphism. As such, there exists a compactly supported 2 -form $\alpha_{\rho}$ so that

$$
\frac{i}{2 \pi} F_{A_{\theta, \rho}}=\alpha_{\rho}+d \beta_{\rho}
$$

with $\beta_{\rho}$ a 1-form on $M_{\theta}$. Since $F_{A_{\theta, \rho}}=d A_{\theta, \rho}$, after possibly modifying $\alpha_{\rho}$, we can take $\beta_{\rho}$ to be $\frac{i}{2 \pi} A_{\theta, \rho}$ on the ALE end of $M_{\theta}$. From Theorem 3.6(3) we know that the connections $A_{\theta, \rho}$ are asymptotically flat of order 1 and that the curvature $F_{A_{\rho}}=O\left(r^{-4}\right)$ as $r \rightarrow \infty$. Then Stokes' formula gives

$$
\int_{M_{\theta}} c_{1}\left(\mathcal{R}_{\rho}\right) c_{1}\left(\mathcal{R}_{\sigma}\right) c_{1}\left(\mathcal{R}_{\tau}\right)=\int_{M_{\theta}} \alpha_{\rho} \wedge \alpha_{\sigma} \wedge \alpha_{\tau}
$$

for all $\rho, \sigma, \tau \in \operatorname{Irr}(G)$. From here, we see that (1.2) is interpreted topologically in terms of triple products

$$
\int_{M_{\theta}}: H_{c}^{2}\left(M_{\theta}, \mathbb{R}\right) \times H_{c}^{2}\left(M_{\theta}, \mathbb{R}\right) \times H_{c}^{2}\left(M_{\theta}, \mathbb{R}\right) \rightarrow \mathbb{R}
$$

with the first Chern classes $c_{1}\left(\mathcal{R}_{\rho}\right)$ thought of as their own images in $H_{c}^{2}\left(M_{\theta}, \mathbb{R}\right)$ under $j^{-1}$.

Therefore, it remains to prove Theorem 1.4 at the level of differential forms. As we already mentioned, the proof uses the Atiyah-Patodi-Singer index theorem for ALE manifolds (6.1). In order to apply it, we need to compute the $\eta$-invariant term that appears in this formula.

Proposition 7.1. Let $G$ be a finite subgroup of $\mathrm{SL}(n, \mathbb{C})$ acting freely on $\mathbb{C}^{n} \backslash\{0\}$. Assume that $X$ is a smooth ALE spin manifold asymptotic to $\mathbb{C}^{n} / G$ and let $(E, A)$ be a asymptotically flat bundle on $X$ whose fiber at infinity is $E_{\infty}$. Then, the $\eta$-invariant for the Dirac operator $D_{E, \delta}$ on $X$ is given by

$$
\eta_{E}=-\frac{2}{|G|} \sum_{\substack{g \in G \\ g \neq I_{n}}} \frac{\chi_{E_{\infty}}(g)}{\sum_{i=0}^{n}(-1)^{i} \chi_{\Lambda^{i} \mathbb{C}^{n}}(g)},
$$

provided $-2 n+1<\delta<0$. In this formula $\chi_{E_{\infty}}$ denotes the character of the representation corresponding to the action of $G$ on the vector space $E_{\infty}$.

Remark 7.2. Note that for any $g \in \mathrm{SL}(n, \mathbb{C}), \sum_{i=0}^{n}(-1)^{i} \chi_{\Lambda^{i} \mathbb{C}^{n}}(g)=\operatorname{det}\left(\operatorname{id}_{\mathbb{C}^{n}}-g\right)$. Since $G$ is chosen to act freely on $\mathbb{C}^{n}, \operatorname{det}\left(I_{n}-g\right) \neq 0$ for all $g \in G \backslash\left\{I_{n}\right\}$, and thus all the denominators in formula (7.1) are non-zero. 
This proposition is a consequence of the Lefschetz fixed-point formula, in the sense that $\eta_{E}$ is the contribution from the fixed locus under the action of $G$ on $\mathbb{C}^{n}$. It can be also proved using the definition of the $\eta$-invariant as the analytic continuation at 0 of the $\eta$-series corresponding to the spectrum of the Dirac operator on the boundary at infinity of the orbifold $\mathbb{C}^{n} / G$. This last approach gives the generalisation of the above formula to the case of non-isolated singularities [11].

Let $G \subset \operatorname{SL}(n, \mathbb{C})$ be a finite subgroup. Then for each $\rho \in \operatorname{Irr}(G)$ we have the decomposition into irreducibles

$$
\Lambda^{i} \mathbb{C}^{n} \otimes \rho=\sum_{\sigma \in \operatorname{Irr}(G)} a_{\rho \sigma}^{(i)} \sigma
$$

with $a_{\rho \sigma}^{(i)} \in \mathbb{N}_{0}$. As in Ito and Nakajima [18], we define

$$
c_{\rho \sigma}:=\sum_{i=0}^{n}(-1)^{i} a_{\rho \sigma}^{(i)}
$$

and set

$$
\tilde{C}:=\left(c_{\rho \sigma}\right)_{\rho, \sigma \in \operatorname{Irr}(G)} \quad \text { and } \quad C:=\left(c_{\rho \sigma}\right)_{\rho, \sigma \in \operatorname{Irr}_{0}(G)} .
$$

Remark 7.3. When $G$ is a finite subgroup of $\operatorname{SL}(2, \mathbb{C})$, the matrix $C$ is the Cartan matrix of the simple Lie algebra corresponding to $G$, while $\tilde{C}$ is its extended version. This is the essence of the classical McKay correspondence [23]. For $n \geq 3$ this matrix has, in general, entries on the diagonal which are not equal to 2, and hence is neither the Cartan matrix associated to a Lie algebra nor is it a generalised Cartan matrix as appearing in the context of Lie algebras.

If $n$ is even, then the virtual representation $\sum_{i=0}^{n}(-1)^{i} \Lambda^{i} \mathbb{C}^{n}$ is self-dual and $C$ is symmetric; otherwise the virtual representation is anti-self-dual and $C$ is anti-symmetric. If $G$ is abelian, as is the case in our situation, then every irreducible representation is one-dimensional and thus $\sum_{\sigma \in \operatorname{Irr}(G)} a_{\rho \sigma}^{(i)}=\operatorname{dim} \Lambda^{i} \mathbb{C}^{n}$. Combined with formula (7.3) this gives

$$
\sum_{\sigma \in \operatorname{Irr}(G)} c_{\rho \sigma}=0
$$

for all $\rho \in \operatorname{Irr}(G)$.

Proof of Theorem 1.4. By Proposition 6.2 we know that index $D_{\mathcal{R}_{\rho} \otimes \mathcal{R}_{\sigma}^{*}, \delta}=0$ for all weights $\delta \in(-5,0)$ and for all $\rho, \sigma \in \operatorname{Irr}(G)$. Thus the index formula (6.1) takes the form

$$
\int_{M_{\theta}} \operatorname{ch}\left(\mathcal{R}_{\rho} \otimes \mathcal{R}_{\sigma}^{*}\right) \hat{A}\left(M_{\theta}\right)=\frac{\eta_{R_{\rho} \otimes R_{\sigma^{*}}}}{2}
$$

Multiplying this equation by the matrix $\widetilde{C}$ yields

$$
\sum_{\rho \in \operatorname{Irr}(G)} c_{\tau \rho} \int_{M_{\theta}} \operatorname{ch}\left(\mathcal{R}_{\rho} \otimes \mathcal{R}_{\sigma}^{*}\right) \hat{A}\left(M_{\theta}\right)=\sum_{\rho \in \operatorname{Irr}(G)} c_{\tau \rho} \frac{\eta_{R_{\rho} \otimes R_{\sigma^{*}}}}{2}
$$

for all $\tau \in \operatorname{Irr}(G)$. The left-hand side of (7.6) can be written as

$$
\sum_{\rho \in \operatorname{Irr}(G)} c_{\tau \rho} \int_{M_{\theta}} \tilde{\operatorname{ch}}\left(\mathcal{R}_{\rho}\right) \tilde{\operatorname{ch}}\left(\mathcal{R}_{\sigma}^{*}\right)
$$




$$
\begin{aligned}
& +\sum_{\rho \in \operatorname{Irr}(G)} c_{\tau \rho} \int_{M_{\theta}} \operatorname{ch}\left(\mathcal{R}_{\rho}\right) \hat{A}\left(M_{\theta}\right)+\sum_{\rho \in \operatorname{Irr}(G)} c_{\tau \rho} \int_{M_{\theta}} \operatorname{ch}\left(\mathcal{R}_{\sigma}^{*}\right) \hat{A}\left(M_{\theta}\right) \\
= & \sum_{\rho \in \operatorname{Irr}(G)} c_{\tau \rho} \int_{M_{\theta}} \tilde{\operatorname{hh}}\left(\mathcal{R}_{\rho}\right) \tilde{\operatorname{ch}}\left(\mathcal{R}_{\sigma}^{*}\right)+\sum_{\rho \in \operatorname{Irr}(G)} c_{\tau \rho} \frac{\eta_{R_{\rho}}}{2}+\sum_{\rho \in \operatorname{Irr}(G)} c_{\tau \rho} \frac{\eta_{R_{\sigma}^{*}}}{2}
\end{aligned}
$$

for all $\tau \in \operatorname{Irr}(G)$. Here we have used the fact that $\hat{A}\left(M_{\theta}\right)=1+\hat{A}_{4}\left(M_{\theta}\right)$ since $M_{\theta}$ is 6 dimensional. By (7.5) the third term in the above expression vanishes. Therefore we can rewrite (7.6) as

$$
\sum_{\rho \in \operatorname{Irr}(G)} c_{\tau \rho} \int_{M_{\theta}} \tilde{\operatorname{ch}}\left(\mathcal{R}_{\rho}\right) \tilde{\operatorname{ch}}\left(\mathcal{R}_{\sigma}^{*}\right)=\sum_{\rho \in \operatorname{Irr}(G)} c_{\tau \rho} \frac{\eta_{R_{\rho} \otimes R_{\sigma^{*}}}}{2}-\sum_{\rho \in \operatorname{Irr}(G)} c_{\tau \rho} \frac{\eta_{R_{\rho}}}{2} .
$$

Taking the characters of (7.2) and summing over $i$ with alternating signs we obtain

$$
\left(\chi_{\mathbb{C}^{3}}(g)-\chi_{\Lambda^{2} \mathbb{C}^{3}}(g)\right) \chi_{\tau}(g)=-\sum_{\rho \in \operatorname{Irr}(G)} c_{\tau \rho} \chi_{\rho}(g),
$$

which gives

$$
\chi_{\tau \otimes \sigma^{*}}(g)=-\sum_{\rho \in \operatorname{Irr}(G)} c_{\tau \rho} \frac{\chi_{\rho \otimes \sigma^{*}}(g)}{\chi_{\mathbb{C}^{3}}(g)-\chi_{\Lambda^{2} \mathbb{C}^{3}}(g)}
$$

for all $g \in G \backslash\{e\}$. Summing over all such $g$ and using formula (7.1) for the $\eta$-invariant, we obtain

$$
-2\left(\delta_{\tau \sigma}-\frac{1}{|G|}\right)=\sum_{\rho \in \operatorname{Irr}(G)} c_{\tau \rho} \eta_{\mathcal{R}_{\rho} \otimes \mathcal{R}_{\sigma}^{*}}
$$

Hence, (7.7) yields

$$
\sum_{\rho \in \operatorname{Irr}(G)} c_{\tau \rho} \int_{M_{\theta}} \tilde{\operatorname{ch}}\left(\mathcal{R}_{\rho}\right) \tilde{\operatorname{ch}}\left(\mathcal{R}_{\sigma}^{*}\right)=-\left(\delta_{\tau \sigma}-\frac{1}{|G|}\right)+\left(\delta_{\tau \rho_{0}}-\frac{1}{|G|}\right)
$$

for all $\tau, \sigma \in \operatorname{Irr}(G)$. For $\tau \in \operatorname{Irr}_{0}(G)$ this becomes

$$
\sum_{\rho \in \operatorname{Irr}(G)} c_{\tau \rho} \int_{M_{\theta}} \tilde{\operatorname{ch}}\left(\mathcal{R}_{\rho}\right) \tilde{\operatorname{ch}}\left(\mathcal{R}_{\sigma}^{*}\right)=-\delta_{\tau \sigma}
$$

Since $\tilde{\operatorname{ch}}\left(\mathcal{R}_{\rho_{0}}\right)=0$, it follows that the matrix $C=\left(c_{\tau \rho}\right)_{\tau, \rho \in \operatorname{Irr}_{0}(G)}$ is invertible and

$$
\int_{M_{\theta}} \tilde{\operatorname{ch}}\left(\mathcal{R}_{\rho}\right) \tilde{\operatorname{ch}}\left(\mathcal{R}_{\sigma}^{*}\right)=-\left(C^{-1}\right)_{\rho \sigma},
$$

for all $\rho, \sigma \in \operatorname{Irr}_{0}(G)$, which is precisely (1.2).

Remark 7.4. Note that formula (7.8) shows that the matrix $C$ is invertible. In the case of a finite subgroup of $\mathrm{SL}(2, \mathbb{C})$ the invertibility of $C$ was a direct consequence of the McKay correspondence, given that $C$ is the Cartan matrix associated to a simply-laced Coxeter-Dynkin diagram [23].

Remark 7.5. From formula (7.9) it can be easily deduced that the set $\left\{\tilde{c h}\left(\mathcal{R}_{\rho}\right): \rho \in \operatorname{Irr}_{0}(G)\right\}$ is linearly independent in $H_{c}^{*}\left(M_{\theta}, \mathbb{R}\right)$. To see this, note that if we had a linear combination $\sum_{\rho \in \operatorname{Irr}_{0}(G)} a_{\rho} \tilde{\operatorname{ch}}\left(\mathcal{R}_{\rho}\right)=0$, then by multiplying with $\tilde{\mathrm{ch}}\left(\mathcal{R}_{\sigma}^{*}\right)$ for $\sigma \in \operatorname{Irr}_{0}(G)$ the left-hand side becomes $-\sum_{\rho \in \operatorname{Irr}_{0}(G)} a_{\rho}\left(C^{-1}\right)_{\rho \sigma}$ and thus all $a_{\rho}$ must vanish. 


\section{Acknowledgments}

A.D. would like to thank Tom Mrowka, Tamás Hausel, Rafe Mazzeo and Mark Stern for useful conversations about different aspects of this work. A.D. was supported by the DFG via SFB/Transregio 71 "Geometric Partial Differential Equations". Parts of this article are the outcome of work undertaken by T.W. while working on his PhD thesis at Imperial College London, supported by European Research Council Grant 247331. T.W. would like to thank his supervisor Simon Donaldson for his support. Both authors would like to thank the anonymous referee of an earlier version of this article for pointing out a way of deriving the multiplicative formula (1.3) from the work of Ito and Nakajima [18].

\section{References}

[1] Atiyah M.F., Patodi V.K., Singer I.M., Spectral asymmetry and Riemannian geometry. I, Math. Proc. Cambridge Philos. Soc. 77 (1975), 43-69.

[2] Bando S., Einstein-Hermitian metrics on noncompact Kähler manifolds, in Einstein Metrics and YangMills Connections (Sanda, 1990), Lecture Notes in Pure and Appl. Math., Vol. 145, Dekker, New York, 1993, 27-33.

[3] Bando S., Kasue A., Nakajima H., On a construction of coordinates at infinity on manifolds with fast curvature decay and maximal volume growth, Invent. Math. 97 (1989), 313-349.

[4] Bartnik R., The mass of an asymptotically flat manifold, Comm. Pure Appl. Math. 39 (1986), 661-693.

[5] Baum P., Fulton W., MacPherson R., Riemann-Roch and topological $K$ theory for singular varieties, Acta Math. 143 (1979), 155-192.

[6] Blichfeldt H.F., Finite collineation groups: with an introduction to the theory of groups of operators and substitution groups, University of Chicago Press, Chicago, 1917.

[7] Bridgeland T., King A., Reid M., The McKay correspondence as an equivalence of derived categories, J. Amer. Math. Soc. 14 (2001), 535-554.

[8] Bühler T., An introduction to the derived category, Notes to a series of lectures given at the Mirror Symmetry Learning Seminar, ETH Zürich, 2007, available at http://xwww.uni-math.gwdg.de/theo/intro-derived. pdf.

[9] Calderbank D.M.J., Gauduchon P., Herzlich M., Refined Kato inequalities and conformal weights in Riemannian geometry, J. Funct. Anal. 173 (2000), 214-255, math.DG/9909116.

[10] Craw A., Ishii A., Flops of $G$-Hilb and equivalences of derived categories by variation of GIT quotient, Duke Math. J. 124 (2004), 259-307, math/0211360.

[11] Degeratu A., Eta-invariants and Molien series for unimodular groups, Ph.D. Thesis, Massachusetts Institute of Technology, 2001.

[12] Degeratu A., Mazzeo R., Fredholm theory for elliptic operators on quasi-asymptotically conical spaces, arXiv:1406.3465.

[13] Gocho T., Nakajima H., Einstein-Hermitian connections on hyper-Kähler quotients, J. Math. Soc. Japan 44 (1992), 43-51.

[14] Gonzalez-Sprinberg G., Verdier J.-L., Construction géométrique de la correspondance de McKay, Ann. Sci. École Norm. Sup. (4) 16 (1983), 409-449.

[15] Hartshorne R., Algebraic geometry, Graduate Texts in Mathematics, Vol. 52, Springer-Verlag, New York Heidelberg, 1977.

[16] Hitchin N., Harmonic spinors, Adv. Math. 14 (1974), 1-55.

[17] Huybrechts D., Fourier-Mukai transforms in algebraic geometry, Oxford Mathematical Monographs, The Clarendon Press, Oxford University Press, Oxford, 2006.

[18] Ito Y., Nakajima H., McKay correspondence and Hilbert schemes in dimension three, Topology 39 (2000), 1155-1191, math.AG/9803120.

[19] Joyce D.D., Compact manifolds with special holonomy, Oxford Mathematical Monographs, Oxford University Press, Oxford, 2000. 
[20] King A.D., Moduli of representations of finite-dimensional algebras, Quart. J. Math. Oxford Ser. (2) 45 (1994), 515-530.

[21] Kronheimer P.B., The construction of ALE spaces as hyper-Kähler quotients, J. Differential Geom. 29 (1989), 665-683.

[22] Kronheimer P.B., Nakajima H., Yang-Mills instantons on ALE gravitational instantons, Math. Ann. 288 (1990), 263-307.

[23] McKay J., Graphs, singularities, and finite groups, in The Santa Cruz Conference on Finite Groups (Univ. California, Santa Cruz, Calif., 1979), Proc. Sympos. Pure Math., Vol. 37, Amer. Math. Soc., Providence, R.I., 1980, 183-186.

[24] Neeman A., Algebraic and analytic geometry, London Mathematical Society Lecture Note Series, Vol. 345, Cambridge University Press, Cambridge, 2007.

[25] Sardo Infirri A.V., Partial resolutions of orbifold singularities via moduli spaces of HYM-type bundles, alg-geom/9610004.

[26] Thomas R.P., Derived categories for the working mathematician, in Winter School on Mirror Symmetry, Vector Bundles and Lagrangian Submanifolds (Cambridge, MA, 1999), AMS/IP Stud. Adv. Math., Vol. 23, Amer. Math. Soc., Providence, RI, 2001, 349-361, math.AG/0001045.

[27] Toen B., K-théorie et cohomologie des champs algébriques, Ph.D. Thesis, Université Paul Sabatier, Toulouse, 1999.

[28] Walpuski T., Gauge theory on $\mathrm{G}_{2}$-manifolds, Ph.D. Thesis, Imperial College London, 2013.

[29] Yau S.S.-T., Yu Y., Gorenstein quotient singularities in dimension three, Mem. Amer. Math. Soc. 105 (1993), viii+88 pages. 